\title{
GEOMETRY OF THE POISSON BOOLEAN MODEL ON A REGION OF LOGARITHMIC WIDTH IN THE PLANE
}

\author{
AMITES DASGUPTA, ${ }^{*}$ \\ RAHUL ROY ${ }^{* * * * *}$ AND \\ ANISH SARKAR, ${ }^{* * * * * *}$ Indian Statistical Institute
}

\begin{abstract}
Consider the region $L=\{(x, y): 0 \leq y \leq C \log (1+x), x>0\}$ for a constant $C>0$. We study the percolation and coverage properties of this region. For the coverage properties, we place a Poisson point process of intensity $\lambda$ on the entire half space $\mathbb{R}_{+} \times \mathbb{R}$ and associated with each Poisson point we place a box of a random side length $\rho$. Depending on the tail behaviour of the random variable $\rho$ we exhibit a phase transition in the intensity for the eventual coverage of the region $L$. For the percolation properties, we place a Poisson point process of intensity $\lambda$ on the region $\mathbb{R}^{2}$. At each point of the process we centre a box of a random side length $\rho$. In the case $\rho \leq R$ for some fixed $R>0$ we study the critical intensity $\lambda_{\mathrm{c}}$ of the percolation on $L$.
\end{abstract}

Keywords: Boolean model; Poisson point process; percolation; coverage

2010 Mathematics Subject Classification: Primary 60K35; 60D05

\section{Introduction}

Let $(X, \lambda, \rho)$ be a Poisson Boolean model on $\mathbb{H}=\mathbb{R}_{+} \times \mathbb{R}$, i.e. $X=\left\{\boldsymbol{x}_{1}, \boldsymbol{x}_{2}, \ldots\right\}$ is a homogeneous Poisson point process on $\mathbb{H}$ with intensity $\lambda$, and, at each point $\boldsymbol{x}_{i}$ we situate the box $\boldsymbol{x}_{i}+\left[0, \rho_{i}\right]^{2}$, where $\left\{\rho_{i}: i \geq 1\right\}$ being a collection of independent and identically distributed (i.i.d.) random variables, with each $\rho_{i}$ having the same distribution as the nonnegative random variable $\rho$ and being independent of the underlying Poisson process. The covered (or occupied) region of this Boolean model is defined as $C=\bigcup_{i \geq 1}\left(\boldsymbol{x}_{i}+\left[0, \rho_{i}\right]^{2}\right)$, while the vacant region is $V=\mathbb{H} \backslash C$. In general, the shapes situated at points of the Poisson process are usually balls of random radius (see, e.g. Stoyan et al. (1987), Hall (1988), and Meester and Roy (1996)); however, for the convenience of writing, we consider boxes instead of balls. It may be easily seen that all our results carry through for the standard case.

For an unbounded connected region $L \subseteq \mathbb{H}$, we say that

- there is occupied percolation in $L$ if $C \cap L$ admits an unbounded connected component with positive probability,

\footnotetext{
Received 30 November 2009; revision received 25 February 2011.

* Postal address: Theoretical Statistics and Mathematics Unit, Indian Statistical Institute, 203 B. T. Road, Kolkata 700108, India. Email address: amites@isical.ac.in

** Postal address: Theoretical Statistics and Mathematics Unit, Indian Statistical Institute, 7 S. J. S. Sansanwal Marg, New Delhi 110016, India.

*** Email address: rahul@isid.ac.in

Supported by a grant from the Department of Science and Technology, Government of India.

**** Email address: anish@isid.ac.in
} 
- there is vacant percolation in $L$ if $V \cap L$ admits an unbounded connected component with positive probability,

and, assuming that the unbounded connected region $L$ is such that it is unbounded along the $x$-axis, i.e. for any $x>0$, there exists $y_{x}$ such that $\left(x, y_{x}\right) \in L$,

- there is eventual coverage of $L$ if, with positive probability, there exists $t>0$ such that $L \cap\{(x, y): x>t\} \subseteq C$.

It is easily seen that there is no equivalence of eventual coverage on the vacant region.

For a nondecreasing function $f:[0, \infty) \rightarrow[0, \infty)$, let

$$
L_{f}=\{(x, y): 0 \leq y \leq f(x)\},
$$

and the critical parameters are defined as

$$
\begin{aligned}
& \lambda_{\mathrm{c}}\left(L_{f}\right)=\inf \left\{\lambda: \text { occupied percolation occurs in } L_{f}\right\}, \\
& \lambda_{\mathrm{c}}^{\star}\left(L_{f}\right)=\sup \left\{\lambda: \text { vacant percolation occurs in } L_{f}\right\}, \\
& \lambda_{\mathrm{e}}\left(L_{f}\right)=\inf \left\{\lambda: \text { there is eventual coverage of } L_{f}\right\} .
\end{aligned}
$$

Although we have not specified it explicitly, in all the above three definitions there is an implicit dependence on the random variable $\rho$ and the underlying space $\mathbb{H}$ on which the Poisson point process is defined.

In addition to these critical parameters for coverage and percolation properties of the region $L_{f}$, we may also define the critical parameter associated with the eventual coverage of the graph of a function. In particular, let $g:[0, \infty) \rightarrow[0, \infty)$ and $l_{g}=\{(x, y): y=g(x)\}$. We say that $l_{g}$ is eventually covered if, with positive probability, there exists $t>0$ such that $l_{g} \cap\{(x, y): x>t\} \subseteq C$. Accordingly, we define the critical parameter as

$$
\lambda_{\mathrm{e}}\left(l_{g}\right)=\inf \left\{\lambda: \text { there is eventual coverage of } l_{g}\right\} .
$$

In this paper our focus will be on the study of the coverage and percolation properties of the region $L_{f}$ and the graph $l_{g}$, where $f(x)$ is of the order of $\log x$ and $g$ is an arbitrary, uniformly continuous function. Our motivation in this work is to show that in a two-dimensional region of width smaller than $\log x$ the percolation properties as well as the coverage properties are the same as those respective properties for a one-dimensional line.

In particular, we show the following result.

Theorem 1.1. Let $f(x)=a \log (1+x)$ for some $a>0$, and let $g:[0, \infty) \rightarrow[0, \infty)$ be $a$ uniformly continuous function. Then the following statements hold.

(i) If $\rho$ is such that, for all large $x$,

$$
\mathrm{P}(\rho>x)=\frac{K_{\rho}+\eta(x)}{x^{2}}
$$

for some $K_{\rho}>0$ and $\eta(x) \rightarrow 0$ as $x \rightarrow \infty$, then

$$
\lambda_{\mathrm{e}}\left(L_{f}\right)=\lambda_{\mathrm{e}}\left(l_{g}\right)=\frac{1}{2 K_{\rho}} .
$$


(ii) If $\rho$ is such that

$$
x^{2} \mathrm{P}(\rho>x) \rightarrow 0 \quad \text { as } x \rightarrow \infty
$$

then

$$
\lambda_{\mathrm{e}}\left(L_{f}\right)=\lambda_{\mathrm{e}}\left(l_{g}\right)=\infty
$$

(iii) If $\rho$ is such that

$$
x^{2} \mathrm{P}(\rho>x) \rightarrow \infty \text { as } x \rightarrow \infty
$$

then

$$
\lambda_{\mathrm{e}}\left(L_{f}\right)=\lambda_{\mathrm{e}}\left(l_{g}\right)=0 .
$$

Theorem 1.2. Let $\rho$ be such that $0<\rho \leq R$ for some fixed $R>0$. For $f:[0, \infty) \rightarrow[0, \infty)$ nondecreasing, the following statements hold.

(i) If $f$ is such that $f(x)=o(\log x)$ as $x \rightarrow \infty$ then $\lambda_{\mathrm{c}}\left(L_{f}\right)=\infty$ and $\lambda_{\mathrm{c}}^{\star}\left(L_{f}\right)=0$.

(ii) If $f$ is such that $\log x=o(f(x))$ as $x \rightarrow \infty$ then $\lambda_{\mathrm{c}}\left(L_{f}\right)=\lambda_{\mathrm{c}}^{\star}\left(L_{f}\right)=\lambda_{\mathrm{c}}\left(\mathbb{R}^{2}\right)=\lambda_{\mathrm{c}}^{\star}\left(\mathbb{R}^{2}\right)$.

(iii) If $f$ is such that $f(x) / \log x \rightarrow a$ as $x \rightarrow \infty$ for some $a>0$ then $\lambda_{\mathrm{c}}\left(L_{f}\right)$ is the unique $\lambda \in\left(\lambda_{\mathrm{c}}\left(\mathbb{R}^{2}\right), \infty\right)$ satisfying $\xi^{\star}(\lambda)=$ a, and $\lambda_{\mathrm{c}}^{\star}\left(L_{f}\right)$ is the unique $\lambda \in\left(0, \lambda_{\mathrm{c}}\left(\mathbb{R}^{2}\right)\right)$ satisfying $\xi(\lambda)=a$, where the vacant and occupied correlation lengths $\xi^{\star}(\lambda)$ and $\xi(\lambda)$ are as defined in Propositions 3.1 and 3.2, respectively.

Remark 1.1. In Theorem 1.2(ii) $\lambda_{\mathrm{c}}\left(\mathbb{R}^{2}\right)$ and $\lambda_{\mathrm{c}}^{\star}\left(\mathbb{R}^{2}\right)$ are the critical intensities for occupied and vacant percolation when the Boolean model is defined by a Poisson point process on the entire plane $\mathbb{R}^{2}$. Although the equality of $\lambda_{\mathrm{c}}\left(\mathbb{R}^{2}\right)$ and $\lambda_{\mathrm{c}}^{\star}\left(\mathbb{R}^{2}\right)$ is known only when the shapes are discs of bounded radius (see Meester and Roy (1996)), the result can be easily extended to shapes which are squares of bounded side lengths. We also require versions of Lemmas 3.3, 4.1 and Theorems 3.5, 3.7, 4.3, 4.4 of Meester and Roy (1996) for shapes which are squares of bounded side lengths. It may be seen that these results follow in a similar manner.

In our first result (Theorem 1.1) we examine $\lambda_{\mathrm{e}}\left(L_{f}\right)$ for $f(x)=a \log (1+x), a>0$, and $\rho$ having a heavy-tailed distribution as given by (1.1). Note that, for any $\rho$ having a tail either thicker or thinner than that given by (1.1), the critical intensity $\lambda_{\mathrm{e}}\left(L_{f}\right)$ is trivial, i.e. $\lambda_{\mathrm{e}}\left(L_{f}\right)=0$ or $\infty$, respectively. In this sense the given tail behaviour (1.1) of $\rho$ is critical for the nontriviality of the model.

Our second result (Theorem 1.2) is similar to the result for the Bernoulli bond percolation on the region $L_{f}$ of Grimmett (1983). In the case of Bernoulli bond percolation on a region $L$ of the two-dimensional square lattice, the critical probability $p_{\mathrm{c}}\left(L_{f}\right)$ of percolation is well studied. Grimmett (1983) showed that the critical probability $p_{\mathrm{c}}(L)$ equals $\frac{1}{2}$, which is the critical probability of percolation on the entire square lattice, whenever the function $f$ grows faster than $\log x$, and $p_{\mathrm{c}}(L)$ equals 1 whenever the function $f$ grows slower than $\log x$. While if $f$ is such that $f(x) \sim a \log x$ for some constant $a>0$ then $p_{\mathrm{c}}\left(L_{f}\right)$ is obtained as the unique solution $p$ of the equation $\xi(1-p)=a$, where $\xi(\cdot)$ is the correlation length of the twodimensional Bernoulli bond percolation process. The method of proof is also similar, although with the vacancy and the occupancy structures not being in a duality relation as in the case of Bernoulli bond percolation, we need to do some extra work.

The analogy of eventual coverage in $L_{f}$ with that of the percolation result is also exhibited in Theorem 1.1, where we study the coverage of a one-dimensional line $l_{g}=\{(x, y): y=$ $g(x)\}$ and show that $\lambda_{\mathrm{e}}\left(L_{f}\right)=\lambda_{\mathrm{e}}\left(l_{g}\right)$ for $g$ and $f$ as given in the statement of the theorem. 
In particular, the percolation properties (vis-à-vis critical parameters) of a region with width smaller than $\log x$ is the same as that of a one-dimensional line. This is also the case vis-à-vis the critical parameters for eventual coverage. For a region of width of the order of $\log x$, the percolation properties are different from the corresponding properties for the line, whereas the coverage properties are similar for both the region and the line.

To complement the above two theorems, we study the behaviour at criticality. It is shown that (i) the occurrence or otherwise of percolation in $L_{f}$ at criticality for $f(x)=O(\log x)$ depends on the higher-order terms of $f$, and (ii) the occurrence or otherwise of eventual coverage of $L_{f}$ depends on the higher-order terms of the tail distribution of $\rho$. These are discussed at the end of the proofs of the theorems in the appropriate sections.

Eventual coverage has been studied for quadrants and octants by Athreya et al. (2004). It is the natural analogue of complete coverage of space in Boolean models. Hall (1988) showed that, for the Boolean model defined on $\mathbb{R}^{d}$, complete coverage occurs, i.e. $C=\mathbb{R}^{d}$ almost surely if and only if $\mathrm{E} \rho^{d}=\infty$. Molchanov and Scherbakov (2003) studied the question of complete coverage for an inhomogeneous Poisson Boolean model. Here we study eventual coverage of the region under the log function. Our method of proof here differs from that of Athreya et al. (2004), in that the events we study are not renewal events and as such we cannot use those properties. Instead a more general Borel-Cantelli lemma is needed for our analysis.

The result on percolation complements the work of Tanemura (1993). Tanemura used a Grimmett and Marstrand method to study continuum percolation on slabs, half-spaces, and other regions of space. In particular, Tanemura showed that $\lambda_{\mathrm{c}}\left(\mathbb{R}^{d}\right)=\lambda_{\mathrm{c}}\left(\mathbb{R}_{+}^{d}\right)$ and $\lambda_{\mathrm{c}}^{\star}\left(\mathbb{R}^{d}\right)=$ $\lambda_{\mathrm{c}}^{\star}\left(\mathbb{R}_{+}^{d}\right)$. We extend these results to further subsets of $\mathbb{R}^{2}$. To this end, we need to develop correlation lengths for both occupied and vacant connectivity functions. Tanemura (1996) used lace expansion techniques to study such connectivity functions in high dimensions.

The rest of the paper is organised as follows. In Section 2 we study the coverage properties and in particular prove Theorem 1.2. In Section 3, for each of the cases of infinite occupied and infinite vacant components, we first derive in Propositions 3.1 and 3.2 the properties of the connectivity functions or correlation lengths by vacant or occupied paths, respectively. These are then used to prove Theorem 1.2 for infinite occupied and infinite vacant components.

\section{Proof of Theorem 1.1}

In order to prove the results, we couple various Poisson Boolean models with the same intensity but different random variables specifying the sizes of the boxes at the Poisson points. It is easy to see that these models can be coupled together on one probability space. Let $C(\tau)$ denote the covered region of the Poisson Boolean model $(X, \lambda, \tau)$. For any $(x, y) \in \mathbb{H}$, we define

$$
A_{(x, y)}(\tau)=\{(x, y) \notin C(\tau)\} .
$$

We first show that $\lambda_{\mathrm{e}}\left(l_{g}\right)=1 / 2 K_{\rho}$ when $\rho$ satisfies (1.1). Since $g$ is uniformly continuous, we can choose $0<\delta<\frac{1}{2}$ such that $|g(x)-g(y)|<\frac{1}{2}$ whenever $x, y>0$ and $|x-y|<\delta$.

Let us set

$$
\rho_{\min }=\max \{\rho-1,0\}
$$

and, for $i \geq 1$,

$$
B_{i}=A_{(i \delta, g(i \delta)-1 / 2)}\left(\rho_{\min }\right), \quad D_{i}=A_{(i, g(i))}(\rho), \quad \text { and } \quad E_{i}=\bigcup_{j=0}^{\lfloor a \log (1+i)\rfloor} A_{(i, j)}\left(\rho_{\min }\right) .
$$


We make the following claims.

(C1) The graph $l_{g}$ is eventually covered if $\mathrm{P}_{\lambda}\left(\limsup _{i \rightarrow \infty} B_{i}\right)=0$.

(C2) The graph $l_{g}$ is not eventually covered if $\mathrm{P}_{\lambda}\left(\limsup _{i \rightarrow \infty} D_{i}\right)=1$.

(C3) The region $L_{f}$ is eventually covered if $\mathrm{P}_{\lambda}\left(\lim \sup _{i \rightarrow \infty} E_{i}\right)=0$.

Since $\{(i, g(i)): i \geq 1\} \subset l_{g}=\{(x, g(x)): x>0\}$, claim (C2) follows trivially. For claims (C1) and (C3), observe that if $(x, y) \in C\left(\rho_{\min }\right)$, by the definition of $\rho_{\min }$, we must have $(x, y)+[0,1]^{2} \subseteq C(\rho)$. Now, if $\left(i \delta, g(i \delta)-\frac{1}{2}\right) \in C\left(\rho_{\min }\right)$, by the above choice of $\delta$, we have $\{(x, g(x)): i \delta \leq x \leq(i+1) \delta\} \subseteq\left(i \delta, g(i \delta)-\frac{1}{2}\right)+[0, \delta] \times[0,1] \subseteq C(\rho)$. Hence, (C1) follows. Noting that $a \log (x+1)-a \log x \leq 1$ whenever $a<x$, a similar argument that is used to prove (C1) will establish (C3).

Let $F_{\tau}$ be the distribution function of $\tau$, i.e. $F_{\tau}(t)=\mathrm{P}(\tau \leq t)$, and let $G_{\tau}(t)=1-F_{\tau}(t)=$ $\mathrm{P}(\tau>t)$.

Lemma 2.1. For any $(x, y) \in \mathbb{H}$ and any random variable $\tau$, we have

$$
\mathrm{P}_{\lambda}\left(A_{(x, y)}(\tau)\right)=\exp \left(-2 \lambda \int_{0}^{x} s G_{\tau}(s) \mathrm{d} s-\lambda x \int_{x}^{\infty} G_{\tau}(s) \mathrm{d} s\right) .
$$

Remark. The probability $\mathrm{P}_{\lambda}\left(A_{(x, y)}(\tau)\right)$ does not depend on $y$, which is not surprising given the stationarity of the underlying process in the $y$-direction.

Proof of Lemma 2.1. Let $N_{x, y}$ denote the set of Poisson points whose associated boxes cover the point $(x, y)$, i.e.

$$
N_{x, y}=\left\{(u, v) \in X:(x, y) \in(u, v)+\left[0, \tau_{u, v}\right]^{2}\right\}
$$

where $\tau_{u, v}$ denotes the (random) size of the box at the Poisson point $(u, v)$. Clearly, $N_{x, y}$ is an inhomogeneous Poisson point process with intensity function given by

$$
\lambda_{x, y}(u, v)=1_{\{0<u<x, v<y\}} \lambda \mathrm{P}(\tau>\max (x-u, y-v)) .
$$

Hence, we have $\mathrm{P}_{\lambda}\left(A_{(x, y)}(\tau)\right)=\mathrm{P}_{\lambda}\left(\left|N_{x, y}\right|=0\right)=\exp \left(-\int_{\mathbb{H}} \lambda_{x, y}(u, v) \mathrm{d} u \mathrm{~d} v\right)$, where $|\cdot|$ denotes the cardinality.

Now

$$
\begin{aligned}
\int_{\mathbb{H}} \lambda_{x, y}(u, v) \mathrm{d} u \mathrm{~d} v \\
\quad=\lambda \int_{0}^{x} \int_{-\infty}^{y} G_{\tau}(\max (x-u, y-v)) \mathrm{d} u \mathrm{~d} v \\
=\lambda \int_{0}^{x} \int_{0}^{\infty} G_{\tau}(\max (s, t)) \mathrm{d} s \mathrm{~d} t \\
=\lambda \int_{0}^{x} \int_{0}^{x} G_{\tau}(\max (s, t)) \mathrm{d} s \mathrm{~d} t+\lambda \int_{0}^{x} \int_{x}^{\infty} G_{\tau}(\max (s, t)) \mathrm{d} s \mathrm{~d} t \\
=2 \lambda \int_{0}^{x} s G_{\tau}(s) \mathrm{d} s+\lambda x \int_{x}^{\infty} G_{\tau}(s) \mathrm{d} s
\end{aligned}
$$

This completes the proof. 
In the following lemma we examine the dependency between the events $A_{\left(x_{1}, y_{1}\right)}(\tau)$ and $A_{\left(x_{2}, y_{2}\right)}(\tau)$.

Lemma 2.2. For $\left(x_{1}, y_{1}\right),\left(x_{2}, y_{2}\right) \in \mathbb{H}$ such that $x_{1}<x_{2}$ and any random variable $\tau$, we have

$$
\mathrm{P}_{\lambda}\left(A_{\left(x_{1}, y_{1}\right)}(\tau) \cap A_{\left(x_{2}, y_{2}\right)}(\tau)\right) \leq \mathrm{P}_{\lambda}\left(A_{\left(x_{1}, 0\right)}(\tau)\right) \mathrm{P}\left(A_{\left(x_{2}-x_{1}, 0\right)}(\tau)\right) .
$$

Proof. Using the notation of the previous lemma, we have

$$
\mathrm{P}_{\lambda}\left(A_{\left(x_{1}, y_{1}\right)}(\tau) \cap A_{\left(x_{2}, y_{2}\right)}(\tau)\right)=\mathrm{P}_{\lambda}\left(\left|N_{x_{1}, y_{1}}\right|=0,\left|N_{x_{2}, y_{2}}\right|=0\right) .
$$

Let $N_{x_{2}, y_{2}}^{\left(x_{1}, y_{1}\right)}$ be the set of Poisson points which lie in the strip $\left(x_{1}, x_{2}\right) \times\left(-\infty, y_{2}\right)$ such that each of their associated boxes covers the point $\left(x_{2}, y_{2}\right)$, i.e.

$$
N_{x_{2}, y_{2}}^{\left(x_{1}, y_{1}\right)}=\left\{(u, v) \in X: x_{1}<u<x_{2}, v<y_{2},\left(x_{2}, y_{2}\right) \in(u, v)+\left[0, \tau_{u, v}\right]^{2}\right\},
$$

where, as in Lemma 2.1, $\tau_{u, v}$ denotes the size of the box at the Poisson point $(u, v)$. Again, $N_{x_{2}, y_{2}}^{\left(x_{1}, y_{1}\right)}$ is an inhomogeneous Poisson point process, and, by stationarity, it has the same distribution as $N_{x_{2}-x_{1}, y_{2}}$. Furthermore, the processes $N_{x_{1}, y_{1}}$ and $N_{x_{2}, y_{2}}^{\left(x_{1}, y_{1}\right)}$ depend on the Poisson points situated on the disjoints sets $(0, x) \times(-\infty, y)$ and $\left(x_{1}, x_{2}\right) \times\left(-\infty, y_{2}\right)$, respectively; thus, they are independent.

Since $\left(x_{1}, x_{2}\right) \times\left(-\infty, y_{2}\right) \subseteq\left(0, x_{2}\right) \times\left(-\infty, y_{2}\right)$, we have $\left|N_{x_{2}, y_{2}}^{\left(x_{1}, y_{1}\right)}\right|=0$ whenever $\left|N_{x_{2}, y_{2}}\right|=0$. Hence, we have

$$
\begin{aligned}
\mathrm{P}_{\lambda}\left(\left|N_{x_{1}, y_{1}}\right|=0,\left|N_{x_{2}, y_{2}}\right|=0\right) & \leq \mathrm{P}_{\lambda}\left(\left|N_{x_{1}, y_{1}}\right|=0,\left|N_{x_{2}, y_{2}}^{\left(x_{1}, y_{1}\right)}\right|=0\right) \\
& =\mathrm{P}_{\lambda}\left(\left|N_{x_{1}, y_{1}}\right|=0\right) \mathrm{P}_{\lambda}\left(\left|N_{x_{2}, y_{2}}^{\left(x_{1}, y_{1}\right)}\right|=0\right) \\
& =\mathrm{P}_{\lambda}\left(\left|N_{x_{1}, y_{1}}\right|=0\right) \mathrm{P}_{\lambda}\left(\left|N_{x_{2}-x_{1}, y_{2}}\right|=0\right) .
\end{aligned}
$$

This completes the proof.

Proposition 2.1. For $\rho$ satisfying (1.1), we have $\mathrm{P}_{\lambda}\left(\limsup _{i \rightarrow \infty} B_{i}\right)=0$ whenever $2 \lambda K_{\rho}>1$.

Proof. The Borel-Cantelli lemma ensures that it suffices to show that $\sum_{i=1}^{\infty} \mathrm{P}_{\lambda}\left(B_{i}\right)<\infty$. Since $2 \lambda K_{\rho}>1$, we can choose $\varepsilon>0$ such that $2 \lambda\left(K_{\rho}-\varepsilon\right)>1$. Also, observe that $G_{\rho_{\min }}(s)=\mathrm{P}\left(\rho_{\min }>s\right)=\mathrm{P}(\rho>s+1)=G(s+1)$. Thus, we have

$$
\begin{aligned}
\mathrm{P}_{\lambda}\left(B_{i}\right) & =\mathrm{P}_{\lambda}\left(A_{(i \delta, g(i \delta))}\left(\rho_{\min }\right)\right) \\
& =\exp \left(-2 \lambda \int_{0}^{i \delta} s G_{\rho_{\min }}(s) \mathrm{d} s-\lambda i \delta \int_{i \delta}^{\infty} G_{\rho_{\text {min }}}(s) \mathrm{d} s\right) \\
& \leq \exp \left(-2 \lambda \int_{0}^{i \delta} s G_{\rho_{\min }}(s) \mathrm{d} s\right) \\
& =\exp \left(-2 \lambda \int_{1}^{i \delta+1}(s-1) G(s) \mathrm{d} s\right) .
\end{aligned}
$$

For $\rho$ satisfying (1.1), we can choose $N$ so large that $|\eta(s)| \leq \varepsilon$ for all $s>N$. Therefore, for any $i$ such that $i \delta>N$, we have

$$
\begin{aligned}
\int_{1}^{i \delta+1}(s-1) G(s) \mathrm{d} s & \geq \int_{N}^{i \delta} s G(s) \mathrm{d} s-\int_{0}^{\infty} G(s) \mathrm{d} s \\
& \geq \int_{N}^{i \delta} \frac{K_{\rho}-\varepsilon}{s} \mathrm{~d} s-\int_{0}^{\infty} G(s) \mathrm{d} s \\
& =\left(K_{\rho}-\varepsilon\right) \log (i \delta)-C_{1},
\end{aligned}
$$


where $C_{1}>0$ is a constant. Therefore, for all large enough $i$, we have

$$
\mathrm{P}_{\lambda}\left(B_{i}\right) \leq C_{2}(i \delta)^{-2 \lambda\left(K_{\rho}-\varepsilon\right)}
$$

for some positive constant $C_{2}$. This completes the proof.

Let $A_{1}=[0,1] \times[-1,1]$, and recursively define $A_{n}=[0, n] \times[-n, n] \backslash A_{n-1}$ for $n \geq 2$. The Boolean model on $\mathbb{H}$ may be constructed by superposing independent Poisson processes on the regions $A_{n}, n \geq 1$. Since $\lim \sup D_{i}$ does not depend upon the realisation of the Poisson processes on any finitely many such $A_{n} \mathrm{~s}$, lim sup $D_{i}$ is a tail event and so $\mathrm{P}_{\lambda}\left(\limsup _{i \rightarrow \infty} D_{i}\right)=$ 0 or 1 .

Lemma 2.3. If $\sum_{i=1}^{\infty} \mathrm{P}_{\lambda}\left(D_{i}\right)=\infty$ then

$$
\mathrm{P}_{\lambda}\left(\limsup _{i \rightarrow \infty} D_{i}\right)=1
$$

Proof. In view of the discussion above we need to show that $\mathrm{P}_{\lambda}\left(\limsup _{i \rightarrow \infty} D_{i}\right)>0$. For this, we use an extension due to Kochen and Stone (see Petrov (2004)) of the second Borel-Cantelli lemma which says that if

$$
\sum_{i=1}^{\infty} \mathrm{P}_{\lambda}\left(D_{i}\right)=\infty \quad \text { and } \quad \liminf _{n} \frac{\sum_{1 \leq i, j \leq n} \mathrm{P}_{\lambda}\left(D_{i} \cap D_{j}\right)}{\left(\sum_{1 \leq i \leq n} \mathrm{P}_{\lambda}\left(D_{i}\right)\right)^{2}}=\alpha,
$$

then

$$
\mathrm{P}_{\lambda}\left(\limsup _{i \rightarrow \infty} D_{i}\right) \geq \frac{1}{\alpha}
$$

Note first that

$$
\sum_{1 \leq i, j \leq n} \mathrm{P}_{\lambda}\left(D_{i} \cap D_{j}\right)=\sum_{i=1}^{n} \mathrm{P}_{\lambda}\left(D_{i}\right)+2 \sum_{i=1}^{n-1} \sum_{j=i+1}^{n} \mathrm{P}_{\lambda}\left(D_{i} \cap D_{j}\right) .
$$

Now the double sum can be estimated as

$$
\begin{aligned}
\sum_{i=1}^{n-1} \sum_{j=i+1}^{n} \mathrm{P}_{\lambda}\left(D_{i} \cap D_{j}\right) & =\sum_{i=1}^{n-1} \sum_{j=i+1}^{n} \mathrm{P}_{\lambda}\left(A_{(i, g(i))}(\rho) \cap A_{(j, g(j))}(\rho)\right) \\
& \leq \sum_{i=1}^{n-1} \sum_{j=i+1}^{n} \mathrm{P}_{\lambda}\left(A_{(i, 0)}(\rho)\right) \mathrm{P}_{\lambda}\left(A_{(j-i, 0)}(\rho)\right) \\
& =\sum_{i=1}^{n-1} \mathrm{P}_{\lambda}\left(A_{(i, 0)}(\rho)\right) \sum_{j-i=1}^{n-i} \mathrm{P}_{\lambda}\left(A_{(j-i, 0)}(\rho)\right) \\
& \leq \sum_{i=1}^{n-1} \mathrm{P}_{\lambda}\left(A_{(i, 0)}(\rho)\right) \sum_{k=1}^{n} \mathrm{P}_{\lambda}\left(A_{(k, 0)}(\rho)\right) \\
& \leq\left(\sum_{k=1}^{n} \mathrm{P}_{\lambda}\left(D_{k}\right)\right)^{2} .
\end{aligned}
$$


This gives

$$
\frac{\sum_{1 \leq i, j \leq n} \mathrm{P}_{\lambda}\left(D_{i} \cap D_{j}\right)}{\left(\sum_{1 \leq i \leq n} \mathrm{P}_{\lambda}\left(D_{i}\right)\right)^{2}} \leq \frac{1}{\sum_{i=1}^{n} \mathrm{P}_{\lambda}\left(D_{i}\right)}+2 \rightarrow 2 \quad \text { as } n \rightarrow \infty,
$$

since, by assumption, $\sum_{i=1}^{n} \mathrm{P}_{\lambda}\left(D_{i}\right) \rightarrow \infty$ as $n \rightarrow \infty$.

Proposition 2.2. For $\rho$ satisfying (1.1), we have $\mathrm{P}_{\lambda}\left(\lim _{\sup _{i \rightarrow \infty}} D_{i}\right)=1$ whenever $2 \lambda K_{\rho}<1$.

Proof. In view of Lemma 2.3, we need to show that $\sum_{i=1}^{\infty} \mathrm{P}_{\lambda}\left(D_{i}\right)=\infty$. Since $2 \lambda K_{\rho}<1$, we can choose $\varepsilon>0$ such that $2 \lambda\left(K_{\rho}+\varepsilon\right)<1$. Again, from the representation of $G(s)(=$ $\mathrm{P}(\rho>s))$, choose $N$ so large that $|\eta(s)|<\varepsilon$ for all $s>N$. Then, we have, for $i>N$,

$$
\int_{0}^{i} s G(s) \mathrm{d} s \leq \int_{0}^{N} s G(s) \mathrm{d} s+\int_{N}^{i} \frac{K_{\rho}+\varepsilon}{s} \mathrm{~d} s=\left(K_{\rho}+\varepsilon\right) \log i+C_{3},
$$

where $C_{3}$ is a suitably chosen constant, not depending on $i$. Also,

$$
i \int_{i}^{\infty} G(s) \mathrm{d} s \leq i \int_{i}^{\infty} \frac{K_{\rho}+\varepsilon}{s^{2}} \mathrm{~d} s=K_{\rho}+\varepsilon=C_{4} \quad \text { (say). }
$$

Therefore, we have

$$
\begin{aligned}
\mathrm{P}_{\lambda}\left(D_{i}\right) & =\mathrm{P}_{\lambda}\left(A_{(i, g(i))}(\rho)\right) \\
& =\exp \left(-2 \lambda \int_{0}^{i} s G(s) \mathrm{d} s-\lambda i \int_{i}^{\infty} G(s) \mathrm{d} s\right) \\
& \geq \exp \left(-2 \lambda\left[\left(K_{\rho}+\varepsilon\right) \log i+C_{3}\right]-\lambda C_{4}\right) \\
& =C_{5} i^{-2 \lambda\left(K_{\rho}+\varepsilon\right)},
\end{aligned}
$$

where $C_{5}=\exp \left(-\lambda\left(2 C_{3}+C_{4}\right)\right)$. This completes the proof.

Propositions 2.1 and 2.2 together prove that, when $\rho$ satisfies (1.1), $\lambda_{\mathrm{e}}\left(l_{g}\right)=1 / 2 K_{\rho}$.

In order to show that $\lambda_{\mathrm{e}}\left(L_{f}\right)=1 / 2 K_{\rho}$, since $l_{g} \subset L_{f}$, take $g=f$, so that we have $\lambda_{\mathrm{e}}\left(L_{f}\right) \geq \lambda_{\mathrm{e}}\left(l_{g}\right)=1 / 2 K_{\rho}$. Alternately, the inequality may also be obtained by a renewal argument, as in Athreya et al. (2004), upon taking $g$ to be a constant function, viz. $g \equiv 0$. Therefore, it is enough to show that, for $2 K_{\rho} \lambda>1, L_{f}$ is eventually covered.

Proposition 2.3. For $\rho$ satisfying (1.1), we have $\mathrm{P}_{\lambda}\left(\limsup _{i \rightarrow \infty} E_{i}\right)=0$ whenever $2 \lambda K_{\rho}>1$.

Proof. We have

$$
\mathrm{P}_{\lambda}\left(E_{i}\right) \leq \sum_{j=0}^{\lfloor a \log (1+i)\rfloor} \mathrm{P}_{\lambda}\left(A_{(i, j)}\left(\rho_{\min }\right)\right) \leq a \log (1+i) \mathrm{P}_{\lambda}\left(A_{(i, 0)}\left(\rho_{\min }\right)\right) .
$$

Using the same arguments as in Proposition 2.1, for $\varepsilon>0$ such that $2 \lambda\left(K_{\rho}-\varepsilon\right)>1$, we have, for all sufficiently large $i$,

$$
\mathrm{P}_{\lambda}\left(E_{i}\right) \leq C_{2} a \log (i+1) i^{-2 \lambda\left(K_{\rho}-\varepsilon\right)},
$$

where $C_{2}$ is as in Proposition 2.1. Therefore, using the Borel-Cantelli lemma, we conclude the result. 
This completes the proof of Theorem 1.1(i). Finally, we show how the above can be used to prove Theorem 1.1(ii) when the tail of $\rho$ is thinner and Theorem 1.1(iii) when the tail is fatter.

Suppose that $\rho$ satisfies (1.2). In this case, we may take $s^{2} G(s)=\eta(s)$, where $\eta(s) \rightarrow 0$ as $s \rightarrow \infty$, i.e. $K_{\rho}=0$ in the representation (1.1) of $\rho$. It is enough to show that, for any $\lambda>0$, $l_{g}$ is not eventually covered, which, by Lemma 2.3 , holds when $\sum_{i=1}^{\infty} \mathrm{P}_{\lambda}\left(D_{i}\right)=\infty$. We first choose $\varepsilon>0$ such that $2 \lambda \varepsilon<1$. Following the same estimates as in Proposition 2.2 we obtain, for all sufficiently large $i$,

$$
\mathrm{P}_{\lambda}\left(D_{i}\right) \geq C_{5} i^{-2 \lambda \varepsilon},
$$

where $C_{5}$ is as in Proposition 2.2. This completes the proof in this case.

Finally, we assume that $\rho$ satisfies (1.3). In this case, we can take $s^{2} G(s)=K(s)$, where $K(s) \rightarrow \infty$ as $s \rightarrow \infty$. Fix any $\varepsilon>0$. It is enough to show that, for $\lambda<\varepsilon, L_{f}$ is eventually covered. To show that $L_{f}$ is eventually covered, we need to show that $\sum_{i=1}^{\infty} \mathrm{P}_{\lambda}\left(E_{i}\right)<\infty$, where $E_{i}$ is as defined earlier. Choose $K$ so large that $\lambda K>1$. Choose $N$ so large that $K(s)>K$ for all $s>N$. Following Proposition 2.3 and the estimates in Proposition 2.1, we have, for all sufficiently large $i, \mathrm{P}_{\lambda}\left(E_{i}\right) \leq C_{6}(\log i+1)(i \delta)^{-\lambda K}$ for some constant $C_{6}>0$.

This completes the proof of Theorem 1.1.

Remark. When $\rho$ satisfies $(1.1)$, at criticality $\left(\lambda=1 /\left(2 K_{\rho}\right)\right)$, both scenarios, eventual coverage and no eventual coverage, are possible. For example, consider the following tail behaviour of $\rho$ :

$$
G(s)=\frac{K_{\rho}}{s^{2}}+\frac{\gamma}{s^{2} \log s} \text { for } s>e,
$$

where $K_{\rho}, \gamma>0$.

The graph $l_{g}$, at criticality, i.e. when $\lambda=\lambda_{\mathrm{e}}\left(l_{g}\right)=1 /\left(2 K_{\rho}\right)$, is eventually covered or not depending on whether $\gamma>K_{\rho}$ or $\gamma \leq K_{\rho}$, respectively. To show this, we need to compute exact orders of the probability of $\mathrm{P}_{\lambda}\left(A_{(x, y)}(\rho)\right)$ under the above assumptions.

Note that

$$
x \int_{x}^{\infty} G(s) \mathrm{d} s=x \int_{x}^{\infty}\left(\frac{K_{\rho}}{s^{2}}+\frac{\gamma}{s^{2} \log s}\right) \mathrm{d} s \rightarrow K_{\rho} \quad \text { as } x \rightarrow \infty .
$$

Also, we have, for $x>e$,

$$
\begin{aligned}
\int_{0}^{x} s G(s) \mathrm{d} s & =\int_{0}^{e} s G(s) \mathrm{d} s+\int_{e}^{x} s G(s) \mathrm{d} s \\
& =\int_{0}^{e} s G(s) \mathrm{d} s+\int_{\mathrm{e}}^{x}\left(\frac{K_{\rho}}{s}+\frac{\gamma}{s \log s}\right) \mathrm{d} s \\
& =C_{7}+K_{\rho}(\log x-1)+\gamma \int_{1}^{\log x} \frac{1}{s} \mathrm{~d} s \\
& =C_{8}+K_{\rho} \log x+\gamma \log \log x,
\end{aligned}
$$

where $C_{7}=\int_{0}^{e} s G(s) \mathrm{d} s$ and $C_{8}=C_{7}-K_{\rho}$. Hence, we have

$$
\mathrm{P}_{\lambda}\left(A_{(x, y)}(\rho)\right)=\exp \left(-\lambda\left(2 C_{8}+\xi(x)\right)\right) \frac{1}{x^{2 \lambda K_{\rho}}(\log x)^{2 \lambda \gamma}},
$$

where $\xi(x) \rightarrow K_{\rho}$ as $x \rightarrow \infty$. 
Using the fact that $2 \lambda K_{\rho}=1$ (at criticality), we obtain $\sum_{i=1}^{\infty} \mathrm{P}_{\lambda}\left(D_{i}\right)=\infty$ if $\gamma \leq K_{\rho}$ and, hence, from Lemma 2.3 we conclude that $\mathrm{P}_{\lambda}\left(\lim \sup _{i \rightarrow \infty} D_{i}\right)=1$; thus, $l_{g}$ is not eventually covered.

On the other hand, using the fact that $G_{\rho_{\min }}(s)=G(s+1)$, we have

$$
\begin{aligned}
\int_{0}^{x} s G_{\rho_{\min }}(s) \mathrm{d} s & \geq \int_{e-1}^{x-1} s G(s+1) \mathrm{d} s \\
& =\int_{e}^{x}(s-1) G(s) \mathrm{d} s \\
& \geq \int_{e}^{x} s G(s) \mathrm{d} s-\int_{0}^{\infty} G(s) \mathrm{d} s \\
& =C_{9}+K_{\rho} \log x+\gamma \log \log x,
\end{aligned}
$$

where $C_{9}$ is a suitably chosen constant. Therefore, we have,

$$
\mathrm{P}_{\lambda}\left(B_{i}\right) \leq \exp \left(-2 \lambda \int_{0}^{i \delta} s G_{\rho_{\min }}(s) \mathrm{d} s\right) \leq \exp \left(-2 \lambda C_{9}\right) \frac{1}{(i \delta)^{2 \lambda K_{\rho}(\log (i \delta))^{2 \lambda \gamma}} .}
$$

Hence, at criticality, for $\gamma>K_{\rho}, \sum_{i=1}^{\infty} \mathrm{P}_{\lambda}\left(B_{i}\right)<\infty$ and the graph $l_{g}$ is eventually covered.

Similar calculations imply that $L_{f}$ is eventually covered if $\gamma>2 K_{\rho}$.

\section{Proof of Theorem 1.2}

\subsection{The case of an infinite occupied component}

We begin by noting that, since the region $L_{f} \subset \mathbb{H}$ and we are assuming that $0<\rho \leq R$ for some fixed $R>0$, it is only the Poisson point process on the half-space $\mathbb{H}$ which determines the percolation properties in the region $L_{f}$.

We also note that, for a Poisson point process $X$ of intensity $\lambda$ on $\mathbb{R}^{2}$ and an independent collection of i.i.d. random variables $\left\{\rho_{i}: i \geq 1\right\}$, the process

$$
Y=\left\{\boldsymbol{x}_{i}+\frac{1}{2}\left(\rho_{i}, \rho_{i}\right): \boldsymbol{x}_{i} \in X\right\}
$$

is again a Poisson point process of intensity $\lambda$. Also, since $\rho_{i} \leq R$ for all $i \geq 1$, the percolation properties in the region $L_{f}$ depend only on the Poisson points and their associated squares placed in the half-space $\{(x, y): y \geq-R\}$. Thus, throughout this section, we assume that $\left\{\boldsymbol{x}_{i}: i \geq 1\right\}$ is a Poisson point process on $\mathbb{R}^{2}$ of intensity $\lambda$ and at $\boldsymbol{x}_{i}$ we place the square $\left[-\rho_{i} / 2, \rho_{i} / 2\right]^{2}$. We also assume without loss of generality that $R=1$, i.e. $\rho \leq 1$.

First we derive properties of the connectivity function for a Poisson Boolean model on $\mathbb{R}^{2}$. Let $B_{n}$ denote the box of side length $2 n$ centred at the origin. We are interested in the probability of the event that there is a vacant path from the origin to $\partial B_{n}$, an event that we write as $\mathbf{0} \stackrel{\star}{\leftrightarrow} \partial B_{n}$. Consider the box $D(\mathbf{0})$ of side length 1 centred at the origin, and consider the event of a vacant path from this box to $\partial B_{n}$, an event we write as $D(\mathbf{0}) \stackrel{\star}{\leftrightarrow} \partial B_{n}$, whose probability we denote by $\beta^{\star}(n)$. We first prove the following result.

Proposition 3.1. We have

$$
\frac{1}{4}(2 n+5)^{-1} \mathrm{e}^{-(n+2) \phi^{\star}(\lambda)} \leq \mathrm{P}_{\lambda}\left(D(\mathbf{0}) \stackrel{\star}{\leftrightarrow} \partial B_{n}\right) \leq 64 \mathrm{e}^{9 \lambda} n \mathrm{e}^{-n \phi^{\star}(\lambda)} .
$$

Also, $-\lim _{m \rightarrow \infty}(1 / m) \log \beta^{\star}(m)=\phi^{\star}(\lambda)$ exists and is a continuous function of $\lambda$. Moreover, $\phi^{\star}(\lambda)=0$ for $\lambda \leq \lambda_{\mathrm{c}}, \phi^{\star}(\lambda)$ is increasing on $\left(\lambda_{\mathrm{c}}, \infty\right)$, and $\phi^{\star}(\lambda) \uparrow \infty$ as $\lambda \uparrow \infty$. As is customary, the vacant correlation length is defined by $\xi^{\star}(\lambda)=1 / \phi^{\star}(\lambda)$. 
Remark. A vacant path from the origin to $\partial B_{n}$ implies that $D(\mathbf{0}) \stackrel{\star}{\leftrightarrow} \partial B_{n}$. On the other hand, a box of side length 3 around the origin which is devoid of Poisson points from $X$ and a vacant path from $D(\mathbf{0})$ to $\partial B_{n}$ implies that $\mathbf{0} \stackrel{\star}{\leftrightarrow} \partial B_{n}$. Using the FKG inequality for the lower bound, we now have

$$
\mathrm{e}^{-9 \lambda} \frac{1}{4}(2 n+5)^{-1} \mathrm{e}^{-(n+2) \phi^{\star}(\lambda)} \leq \mathrm{P}_{\lambda}\left(\mathbf{0} \stackrel{\star}{\leftrightarrow} \partial B_{n}\right) \leq 64 \mathrm{e}^{9 \lambda} n \mathrm{e}^{-n \phi^{\star}(\lambda)} .
$$

Proof of Proposition 3.1. We put boxes of side lengths 1 centred at the integer points of $\partial B_{m+2}$. We note that if there is a vacant path from $D(\mathbf{0})$ to $\partial B_{m+n+2}$ then there is a vacant path from $D(\boldsymbol{0})$ to $\partial B_{m}$ which lies completely in the box $B_{m}$ and a vacant path from $D(\boldsymbol{x})$, where $D(\boldsymbol{x})$ is a box of side length 1 around some $\boldsymbol{x} \in \partial B_{m+2}$ with integer coordinates, to the boundary of a box of side length $2 n$ around this box $D(\boldsymbol{x})$ which lies completely in the annular region $B_{m+n+2} \backslash B_{m+2}$. Since these two events depend on the Poisson points of disjoint regions (viz. int $B_{m+1}$ and $B_{m+n+3} \backslash B_{m+1}$, respectively), by the independence properties of the Poisson process we have, considering all such $D(\boldsymbol{x})$ with $\boldsymbol{x}$ having integer coordinates situated on $\partial B_{m+2}$,

$$
\begin{aligned}
& \beta^{\star}(m+n+2) \leq \beta^{\star}(m) \sum_{x \in \partial B_{m+2}} \beta^{\star}(n), \\
& \beta^{\star}(m+n+2) \leq \beta^{\star}(m) 4(2(m+2)+1) \beta^{\star}(n),
\end{aligned}
$$

i.e.

$$
\log \beta^{\star}(m+n+2) \leq \log \beta^{\star}(m)+\log \beta^{\star}(n)+\log (2(m+2)+1)+\log 4,
$$

which we write for convenience as

$$
\log \beta^{\star}(m+n+2) \leq \log \beta^{\star}(m)+\log \beta^{\star}(n)+g_{1}(m),
$$

where $g_{1}(m)=\log (2 m+5)+\log 4$. Thus, we have an inequality of the form

$$
x_{m+n+2} \leq x_{m}+x_{n}+h_{m} \quad \text { with } \quad \frac{h_{m}}{m} \rightarrow 0 \quad \text { as } m \rightarrow \infty,
$$

which from the generalized subadditive inequality (see Appendix II of Grimmett (1999)) yields

$$
\lim _{m} \frac{\log \beta^{\star}(m)}{m} \text { exists and equals }-\phi^{\star}(\lambda) \text { (say), }
$$

and

$$
\log \beta^{\star}(m) \geq-(m+2) \phi^{\star}(\lambda)-g_{1}(m) .
$$

Conversely, we consider events which imply a vacant path from $D(\mathbf{0})$ to $\partial B_{m+n}$. Writing $D(\boldsymbol{x})=\left[x_{1}-\frac{1}{2}, x_{1}+\frac{1}{2}\right] \times\left[x_{2}-\frac{1}{2}, x_{2}+\frac{1}{2}\right]$, consider the collection $\mathscr{D}_{m}=\{D(\boldsymbol{x}): \boldsymbol{x}$ is such that $\boldsymbol{x}$ has integer coordinates and $x_{i}= \pm m$ for some $\left.i\right\}$. Let $U_{\boldsymbol{x}}=\{D(\mathbf{0}) \stackrel{\star}{\leftrightarrow} D(\boldsymbol{x})\}$ for some $\boldsymbol{x}$ with $D(\boldsymbol{x}) \in \mathscr{D}_{m}$. Without loss of generality, let $\boldsymbol{x}$ be such that $x_{1}=m$. Consider also the event $V_{\boldsymbol{x}}$ of a vacant path from $D(\boldsymbol{x})$ to $\partial B_{n}(\boldsymbol{x}) \cap\left\{x_{1}=m+n\right\}$. If in addition we consider a box of side length 3 around $x$ and require it to be empty, then these three decreasing events imply a vacant path from $D(\mathbf{0})$ to $\partial B_{m+n}$. By the FKG inequality,

$$
\beta^{\star}(m+n) \geq \mathrm{P}_{\lambda}\left(U_{\boldsymbol{x}}\right) \mathrm{e}^{-9 \lambda} \mathrm{P}_{\lambda}\left(V_{\boldsymbol{x}}\right) .
$$

Now a vacant path from $D(\mathbf{0})$ to $\partial B_{n}$ implies the union of vacant paths in fixed directions, and these probabilities in fixed directions are the same by symmetry. Let us define $\gamma^{\star}(n)=$ $\mathrm{P}_{\lambda}\left(D(\mathbf{0}) \stackrel{\star}{\leftrightarrow} \partial B_{n}\right.$ in a given direction). So $\mathrm{P}_{\lambda}\left(V_{\boldsymbol{x}}\right)=\gamma^{\star}(n) \leq \beta^{\star}(n) \leq 4 \gamma^{\star}(n)$. On the other 
hand,

$$
\beta^{\star}(m) \leq \mathrm{P}_{\lambda}\left(\bigcup_{\boldsymbol{x} \in \partial B_{m}} U_{\boldsymbol{x}}\right) \leq \sum_{\boldsymbol{x} \in \partial B_{m}} \mathrm{P}_{\lambda}\left(U_{\boldsymbol{x}}\right),
$$

where the union and the sum are over the integer points on $\partial B_{m}$. Considering the maximum over integer points $\boldsymbol{x}$ in fixed directions we see that there is an $\boldsymbol{x}$ so that $\mathrm{P}_{\lambda}\left(U_{\boldsymbol{x}}\right) \geq \beta^{\star}(m) /\left|\partial B_{m}\right|$, where $\left|\partial B_{m}\right|$ counts integer points on $\partial B_{m}$. Using this and $\mathrm{P}_{\lambda}\left(V_{\boldsymbol{x}}\right)=\gamma^{\star}(n) \geq \beta^{\star}(n) / 4,(3.3)$ gives

$$
\beta^{\star}(m+n) \geq \frac{\mathrm{e}^{-9 \lambda} \beta^{\star}(m) \beta^{\star}(n)}{4\left|\partial B_{m}\right|} .
$$

Now taking

$$
x_{n}=-\log \beta^{\star}(n)+\log (64)+\log n+9 \lambda,
$$

manipulations as in Theorem 6.10 of Grimmett (1999) yield

$$
x_{m+n} \leq x_{m}+x_{n} .
$$

The standard subadditive inequality limit result now implies that

$$
\lim _{m \rightarrow \infty} \frac{x_{m}}{m} \text { exists and equals } \inf _{m \geq 1} \frac{x_{m}}{m} .
$$

Since $(\log (64)+\log m+9 \lambda) / m \rightarrow 0$, we have

$$
\lim _{m \rightarrow \infty} \frac{x_{m}}{m}=\lim _{m \rightarrow \infty} \frac{-\log \beta^{\star}(m)}{m}=\phi^{\star}(\lambda)
$$

and

$$
\log \beta^{\star}(m) \leq-m \phi^{\star}(\lambda)+\log (64)+\log (m)+9 \lambda .
$$

Inequalities (3.2) and (3.4) establish the bounds in (3.1).

We now recall that $\beta^{\star}(m)$ is also a function of $\lambda$, so if we can argue that $\beta^{\star}(m)$ is a continuous function of $\lambda$ for fixed $m$ then inequalities (3.2) and (3.4) give uniform convergence on compact sets, and, hence, $\phi^{\star}(\lambda)$ will be continuous. As $\lambda$ increases the probability $\mathrm{P}_{\lambda}\left(D(\mathbf{0}) \stackrel{\star}{\leftrightarrow} \partial B_{m}\right)$ decreases. Thus, considering the superposition of two independent Poisson processes with intensities $\lambda-\varepsilon$ and $2 \varepsilon$, a simple coupling argument gives

$$
\begin{aligned}
\mathrm{P}_{\lambda-\varepsilon}\left(D(\mathbf{0}) \stackrel{\star}{\leftrightarrow} \partial B_{m}\right) \mathrm{e}^{-2 \varepsilon \operatorname{Vol}\left(B_{m+1}\right)} & \leq \mathrm{P}_{\lambda+\varepsilon}\left(D(\mathbf{0}) \stackrel{\star}{\leftrightarrow} \partial B_{m}\right) \\
& \leq \mathrm{P}_{\lambda-\varepsilon}\left(D(\mathbf{0}) \stackrel{\star}{\leftrightarrow} \partial B_{m}\right) .
\end{aligned}
$$

Thus,

$$
\begin{aligned}
\mathrm{P}_{\lambda-\varepsilon} & \left(D(\mathbf{0}) \stackrel{\star}{\leftrightarrow} \partial B_{m}\right)-\mathrm{P}_{\lambda-\varepsilon}\left(D(\mathbf{0}) \stackrel{\star}{\leftrightarrow} \partial B_{m}\right) \mathrm{e}^{-2 \varepsilon \operatorname{Vol}\left(B_{m+1}\right)} \\
& \geq \mathrm{P}_{\lambda-\varepsilon}\left(D(\mathbf{0}) \stackrel{\star}{\leftrightarrow} \partial B_{m}\right)-\mathrm{P}_{\lambda+\varepsilon}\left(D(\mathbf{0}) \stackrel{\star}{\leftrightarrow} \partial B_{m}\right) \\
& \geq 0,
\end{aligned}
$$

leading to the continuity of $\mathrm{P}_{\lambda}\left(D(\mathbf{0}) \stackrel{\star}{\leftrightarrow} \partial B_{m}\right)$. Hence, $\phi^{\star}(\lambda)$ is a continuous function of $\lambda$.

Now

$$
\begin{aligned}
\theta^{\star}(\lambda) & =\mathrm{P}_{\lambda} \text { (there is an unbounded vacant component containing the origin) } \\
& =\lim _{n \rightarrow \infty} \mathrm{P}_{\lambda}\left(\mathbf{0} \stackrel{\star}{\leftrightarrow} \partial B_{n}\right),
\end{aligned}
$$


and here $\lambda<\lambda_{\mathrm{c}}^{\star}$ implies that $\theta^{\star}(\lambda)>0$. From this and the right-hand side of inequality (3.1), we obtain $\phi^{\star}(\lambda)=0$ for $\lambda<\lambda_{\mathrm{c}}^{\star}$. Continuity gives $\phi^{\star}\left(\lambda_{\mathrm{c}}\right)=0$. On the other hand, if $\lambda>\lambda_{\mathrm{c}}^{\star}$ then, by Theorem 4.3 and Lemma 4.1 of Meester and Roy (1996), $\mathrm{P}_{\lambda}(d(V) \geq a) \leq C_{1} \mathrm{e}^{-a C_{2}}$ for positive constants $C_{1}$ and $C_{2}$, where $d(V)$ denotes the diameter of the vacant component of the origin. This along with the left-hand side of inequality (3.1) implies that $\phi^{\star}(\lambda)>0$ if $\lambda>\lambda_{\mathrm{c}}^{\star}$.

Finally, we want to show that, for $\lambda>\lambda_{\mathrm{c}}, \phi^{\star}(\lambda)$ is increasing and goes to $\infty$ as $\lambda \rightarrow \infty$. For this, we approximate the Poisson process by independent Bernoulli random variables while taking care of the random radius by discretization as follows. Consider the Bernoulli percolation model on $\mathbb{Z}^{2} \times\{1, \ldots, k\}$. Let $\boldsymbol{q}=\left(q_{1}, \ldots, q_{k}\right)$ for $0 \leq q_{1}, \ldots, q_{k} \leq 1$, and let $\mathrm{P}_{\boldsymbol{q}}$ be the product measure on $\{0,1\}^{\mathbb{Z}^{2} \times\{1, \ldots, k\}}$ with marginals given by

$$
\mathrm{P}\left(\omega((u, v, j)=0)=q_{j}\right)=1-\mathrm{P}(\omega((u, v), j)=1) \quad \text { for }(u, v) \in \mathbb{Z}^{2} \text { and } 1 \leq j \leq k .
$$

Let $A$ be a decreasing event which depends on the configuration of a finite subset of $\mathbb{Z}^{2} \times$ $\{1, \ldots, k\}$ in this model. For $\gamma>1$, taking $h(\boldsymbol{q})=\mathrm{P}_{\boldsymbol{q}}(A)$ and $\boldsymbol{q}(\gamma)=\left(q_{1}^{\gamma}, \ldots, q_{k}^{\gamma}\right)$, we claim that

$$
h(\boldsymbol{q}(\gamma)) \leq h(\boldsymbol{q})^{\gamma} .
$$

Indeed, imitating the proof of Theorem 2.38 of Grimmett (1999), we note that, for $A$ depending on only one vertex $(u, v, j)$ (say),

$$
h(\boldsymbol{q})=q_{j} \text { or } 1 \text { depending on whether } A=\{0\} \text { or }\{0,1\} \text {, respectively, }
$$

and inequality (3.7) holds trivially.

Suppose now that the result holds whenever $A$ depends only on $l$ vertices for $l \leq m-1$. For $A$ depending on $m$ vertices $\left(u_{1}, v_{1}, j_{1}\right), \ldots,\left(u_{m}, v_{m}, j_{m}\right)$, we have

$$
\begin{aligned}
\mathrm{P}_{\boldsymbol{q}(\gamma)}(A)= & \mathrm{P}_{\boldsymbol{q}(\gamma)}\left(A \mid \omega\left(u_{m}, v_{m}, j_{m}\right)=0\right) q_{j_{m}}^{\gamma} \\
& +\mathrm{P}_{\boldsymbol{q}(\gamma)}\left(A \mid \omega\left(u_{m}, v_{m}, j_{m}\right)=1\right)\left(1-q_{j_{m}}^{\gamma}\right) \\
\leq & \mathrm{P}_{\boldsymbol{q}}\left(A \mid \omega\left(u_{m}, v_{m}, j_{m}\right)=0\right)^{\gamma} q_{j_{m}}^{\gamma} \\
& +\mathrm{P}_{\boldsymbol{q}}\left(A \mid \omega\left(u_{m}, v_{m}, j_{m}\right)=1\right)^{\gamma}\left(1-q_{j_{m}}^{\gamma}\right) \\
\leq & \left(\mathrm{P}_{\boldsymbol{q}}\left(A \mid \omega\left(u_{m}, v_{m}, j_{m}\right)=0\right) q_{j_{m}}\right. \\
& \left.+\mathrm{P}_{\boldsymbol{q}}\left(A \mid \omega\left(u_{m}, v_{m}, j_{m}\right)=1\right)\left(1-q_{j_{m}}\right)\right)^{\gamma} \\
= & \mathrm{P}_{\boldsymbol{q}}(A)^{\gamma},
\end{aligned}
$$

where the last inequality follows on noting that, $A$ being a decreasing event,

$$
\mathrm{P}_{\boldsymbol{q}}\left(A \mid \omega\left(u_{m}, v_{m}, j_{m}\right)=0\right) \geq \mathrm{P}_{\boldsymbol{q}}\left(A \mid \omega\left(u_{m}, v_{m}, j_{m}\right)=1\right)
$$

and from the inequality $x^{\gamma} q^{\gamma}+y^{\gamma}\left(1-q^{\gamma}\right) \leq(x q+y(1-q))^{\gamma}$ for $x \geq y \geq 0$.

Now let $l_{1} \leq l_{2} \leq \cdots$ be nonnegative integers, and consider positive-valued discrete random variables $\rho_{k}, k \geq 1$, defined on the same probability space such that $\rho_{k}$ takes values $r_{k, 1}, \ldots, r_{k, l_{k}}$ with probabilities $\alpha_{k, 1}, \ldots, \alpha_{k, l_{k}}$, respectively. Also, assume that $\rho_{i} \geq \rho_{i+1}$ and $\rho_{k}$ converges almost surely to $\rho$ as $k \rightarrow \infty$.

We approximate the Boolean model $\left(X, \lambda, \rho_{k}\right)$ by a Bernoulli model on $(\delta \mathbb{Z})^{2} \times\left\{1, \ldots, l_{k}\right\}$ by declaring the vertex $(u, v, j)$ to be open if and only if there is at least one Poisson point 
situated in $(u, v)+[-\delta / 2, \delta / 2)^{2}$ with the associated box being of length $r_{k, j}$. Since the Boolean model $\left(X, \lambda, \rho_{k}\right)$ may be written as a superposition of independent models $\left(X_{k, i}, \lambda \alpha_{k, i}, r_{i}\right), i=$ $1, \ldots, l_{k}$, we obtain a Bernoulli percolation model on $(\delta \mathbb{Z})^{2} \times\left\{1, \ldots, l_{k}\right\}$ with associated probability measure $\mathrm{P}_{\boldsymbol{q}}$ for $\boldsymbol{q}=\left(\mathrm{e}^{-\delta^{2} \lambda \alpha_{k, 1}}, \ldots, \mathrm{e}^{-\delta^{2} \lambda \alpha_{k, l_{k}}}\right)$.

Let

$$
\begin{array}{r}
V_{\delta}^{k}=\left\{(x, y) \in \mathbb{R}^{2}:(x, y) \notin(u, v)+\left[-\frac{1}{2} r_{k, j}, \frac{1}{2} r_{k, j}\right]^{2} \text { for }(u, v, j) \text { with }(u, v) \in(\delta \mathbb{Z})^{2}\right. \\
\text { and } \left.\omega(u, v, j)=1 \text { for any } j=1, \ldots, l_{k}\right\},
\end{array}
$$

i.e. the complement of the projection on $\mathbb{R}^{2}$ of the occupied region in each of the hyperplanes of $(\delta \mathbb{Z})^{2} \times\left\{1, \ldots, l_{k}\right\}$. Let

$$
\begin{array}{r}
A_{\delta}^{k}=\left\{V_{\delta}^{k} \text { has a connected component } W \text { such that } D(\mathbf{0}) \cap W\right. \text { is nonempty } \\
\text { and } \left.W \cap \partial B_{n} \text { is nonempty }\right\} .
\end{array}
$$

Then, as $\delta \rightarrow 0, V_{\delta}^{k} \cap B_{n} \rightarrow V^{k} \cap B_{n}$, where $V^{k}$ is the vacant region under $\left(X, \lambda, \rho_{k}\right)$ on $\mathbb{R}^{2}$ and $A_{\delta}^{k} \rightarrow A^{k}$, where $A^{k}$ is the required event $D(\mathbf{0}) \stackrel{\star}{\leftrightarrow} \partial B_{n}$ under the Boolean model $\left(X, \lambda, \rho_{k}\right)$ and whose probability now satisfies $\mathrm{P}_{\gamma \lambda}\left(A^{k}\right) \leq \mathrm{P}_{\lambda}\left(A^{k}\right)^{\gamma}$. By standard arguments we can make $k \rightarrow \infty$ to obtain $\mathrm{P}_{\gamma \lambda}\left(D(\mathbf{0}) \stackrel{\star}{\leftrightarrow} \partial B_{n}\right) \leq \mathrm{P}_{\lambda}\left(D(\mathbf{0}) \stackrel{\star}{\leftrightarrow} \partial B_{n}\right)^{\gamma}$, which after taking logarithms gives

$$
-\gamma \frac{1}{n} \log \mathrm{P}_{\lambda}\left(D(\mathbf{0}) \stackrel{\star}{\leftrightarrow} \partial B_{n}\right) \leq-\frac{1}{n} \log \mathrm{P}_{\gamma \lambda}\left(D(\mathbf{0}) \stackrel{\star}{\leftrightarrow} \partial B_{n}\right) .
$$

As we make $n \rightarrow \infty$, we obtain $\phi^{\star}(\gamma \lambda) \geq \gamma \phi^{\star}(\lambda)>\phi^{\star}(\lambda)$ since $\gamma>1$. This shows that $\phi^{\star}(\lambda)$ is increasing for $\lambda$ above $\lambda_{\mathrm{c}}^{\star}$ and goes to $\infty$ as $\lambda \rightarrow \infty$. This completes the proof of Proposition 3.1.

Recall that $\phi^{\star}(\lambda)=1 / \xi^{\star}(\lambda)$, and note that the results in Theorem 1.2(iii) are stated in this notation. Note also that, as stated in Remark 1.1, in two dimensions we have $\lambda_{\mathrm{c}}=\lambda_{\mathrm{c}}^{\star}$.

Proof of Theorem 1.2(iii) for an infinite occupied component. Suppose that $\lambda>\lambda_{\mathrm{c}}$ and $a<$ $\xi^{\star}(\lambda)$. Then we want to show that $L_{f}$ almost surely contains no unbounded occupied cluster. Let $\lambda_{a}$ be the solution of $\xi^{\star}(\lambda)=a$. Note that over $\left(\lambda_{c}^{\star}, \infty\right), \xi^{\star}(\lambda)$ is decreasing; thus, $a<\xi^{\star}(\lambda)$ implies that $\lambda<\lambda_{a}$. Choosing $\delta>0$ such that $(1+\delta) a<\xi^{\star}(\lambda)$, we define $\boldsymbol{w}_{k}=\left(k^{1+\delta}, 0\right)$. Let $B_{k}$ be the smallest square with $\boldsymbol{w}_{k}$ in the middle of the lower side with the upper side just above the curve $v=f(u)$. By our assumption, $f(u) / \log u \rightarrow a$; hence, the side length $l_{k}$ of $B_{k}$ satisfies

$$
l_{k}=a(1+o(1)) \log k^{1+\delta} \quad \text { as } k \rightarrow \infty .
$$

Now $B_{k}$ has side length $l_{k}$ and centre $\boldsymbol{w}_{k}+\left(0, l_{k} / 2\right)$. Let $A_{k}$ be the event that there is a vacant path from the top edge to the bottom edge of $B_{k}$. By the FKG inequality,

$$
\mathrm{P}_{\lambda}\left(A_{k}\right) \geq\left\{\frac{1}{4} \mathrm{P}_{\lambda}\left(D(\mathbf{0}) \stackrel{\star}{\leftrightarrow} \partial B_{k}\left(\frac{1}{2} l_{k}\right)\right)\right\}^{2} \mathrm{e}^{-9 \lambda} .
$$

However,

$$
\mathrm{P}_{\lambda}\left(D(\mathbf{0}) \stackrel{\star}{\leftrightarrow} \partial B_{k}\left(\frac{1}{2} l_{k}\right)\right) \approx \mathrm{e}^{-l_{k}\left(2 \xi^{\star}(\lambda)\right)^{-1}} \quad \text { as } k \rightarrow \infty,
$$

by (3.1), where ' $\approx$ ' denotes equality in the limit after taking the logarithm and dividing by $l_{k}$. Hence, $\mathrm{P}_{\lambda}\left(A_{k}\right) \geq \frac{1}{16} \mathrm{e}^{-9 \lambda} k^{-(1+o(1))(1+\delta) a / \xi^{\star}(\lambda)}$ as $k \rightarrow \infty$, i.e. $\sum \mathrm{P}_{\lambda}\left(A_{k}\right)=\infty$ since $(1+$ $\delta) a<\xi^{\star}(\lambda)$. On the other hand, for large $k$, the squares $B_{k}$ are separated by more than 
twice the maximum of the sides of the Boolean squares (assumed that $R=1$ here); hence, the configurations inside the $B_{k} \mathrm{~s}$ are independent for large $k$. Thus, $A_{k}$ occurs infinitely often almost surely.

Secondly, suppose that $\lambda>\lambda_{\mathrm{c}}$ and $a>\xi^{\star}(\lambda)$. We want to show that $L_{f}$ almost surely contains an infinite occupied cluster. Choose $\alpha$ such that $a>\alpha>\xi^{\star}(\lambda)$, and let $D_{k}$ be the box with centre $(k, 0)$ and side length $2 \alpha \log k$. For large values of $k, D_{k}$ lies strictly beneath the curve $v=f(u)$. Let $E_{k}$ be the event that $(k, 0)$ is joined by a vacant path to $\partial D_{k}$. From (3.1) we have

$$
\mathrm{P}_{\lambda}\left(E_{k}\right)=\mathrm{P}_{\lambda}(\mathbf{0} \stackrel{\star}{\leftrightarrow} \partial B(\alpha \log k)) \leq \mathrm{P}_{\lambda}(D(\mathbf{0}) \stackrel{\star}{\leftrightarrow} \partial B(\alpha \log k)) \approx k^{-\alpha / \xi^{\star}(\lambda)}
$$

as $k \rightarrow \infty$. This gives $\sum \mathrm{P}_{\lambda}\left(E_{k}\right)<\infty$ from the assumption that $\alpha>\xi^{\star}(\lambda)$. Therefore, there exists an $M$ such that

$$
\mathrm{P}_{\lambda}\left(\bigcup_{k \geq M} E_{k}\right)<\frac{1}{2} .
$$

However, if none of the events $\left\{E_{k}, k \geq M\right\}$ occurs then a vacant path cannot join $f(u)$ and the $x$-axis, and $L_{f}$ almost surely contains an infinite open cluster.

Combining the two steps, this proves that $\lambda_{\mathrm{c}}\left(L_{f}\right)$ is the unique solution in $\left(\lambda_{\mathrm{c}}\left(\mathbb{R}^{2}\right), \infty\right)$ of $\xi^{\star}(\lambda)=a$.

Proof of Theorem 1.2(i) and (ii) for an infinite occupied component. In (i), $\lambda_{\mathrm{c}}\left(L_{f}\right)=\infty$ follows from the fact that the solution of $\xi^{\star}(\lambda)=a$ goes to $\infty$ as $a \downarrow 0$. Similarly, in (ii), $\lambda_{\mathrm{c}}\left(L_{f}\right)=\lambda_{\mathrm{c}}\left(\mathbb{R}^{2}\right)$ follows from the fact that the solution of $\xi^{\star}(\lambda)=a$ goes to $\lambda_{\mathrm{c}}\left(\mathbb{R}^{2}\right)$ as $a \uparrow \infty$.

\subsection{The case of an infinite vacant component}

Again, we first derive properties of the connectivity function. When we consider the event that there is an occupied path from the origin to $\partial B_{n}$, much of the argument remains the same, except that now some of the events become increasing events.

First of all, there exists a $t>0$ such that $\mathrm{P}(\rho>2 t)>0$ (as before $\rho \leq 1$ ). We divide the square of side three around $\mathbf{0}$ into $\left[9 / t^{2}\right]$ many small squares of side $t$ and consider the event that in each smaller square there is at least one Poisson point with associated $\rho>2 t$. If an occupied path from $D(\mathbf{0})$ to $\partial B_{m}$ also exists then these two events imply that $\mathbf{0} \leftrightarrow \partial B_{m}$, which is our notation for an occupied path from one to the other. Thus, using the FKG inequality, it is enough to work with $\mathrm{P}\left(D(\mathbf{0}) \leftrightarrow \partial B_{m}\right)=\beta(m)$, and parallel arguments lead to the existence of the limit

$$
\phi(\lambda)=\lim -\frac{1}{m} \log \mathrm{P}_{\lambda}\left(D(\mathbf{0}) \leftrightarrow \partial B_{m}\right),
$$

along with inequalities similar to (3.2) and (3.4). There is a small difference in the analogue of (3.4). We are considering occupied paths here, so in the analogue of (3.3) we can fill a box of side length 1 around $\boldsymbol{x}$ with probability $\left(1-\mathrm{e}^{-\lambda t^{2} \mathrm{P}(\rho>2 t)}\right)^{9 / t^{2}}$, where $t$ is as given above, leading to the following result.

Proposition 3.2. We have

$$
\frac{1}{4}(2 n+5)^{-1} \mathrm{e}^{-(n+2) \phi(\lambda)} \leq \mathrm{P}_{\lambda}\left(D(\mathbf{0}) \leftrightarrow \partial B_{n}\right) \leq 64 n\left(1-\mathrm{e}^{-\lambda t^{2} \mathrm{P}(\rho>2 t)}\right)^{-9 / t^{2}} \mathrm{e}^{-n \phi(\lambda)} .
$$

The limit of $-(1 / m) \log \beta(m)=\phi(\lambda)$ is a continuous function of $\lambda$. Moreover, $\phi(\lambda)=0$ for $\lambda \geq \lambda_{\mathrm{c}}, \phi(\lambda)$ is decreasing on $\left(0, \lambda_{\mathrm{c}}\right)$, and $\phi(\lambda) \uparrow \infty$ as $\lambda \downarrow 0$. As is customary, the occupied correlation length is defined by $\xi(\lambda)=1 / \phi(\lambda)$. 
Proof. Continuity of $\phi(\lambda)$ follows as before from the continuity of $\mathrm{P}_{\lambda}\left(D(\mathbf{0}) \leftrightarrow \partial B_{n}\right)$ in $\lambda$ for each fixed $n$, which can be proved by noting that in inequalities (3.5) and (3.6) we can substitute $\mathrm{P}_{\lambda \pm \varepsilon}\left\{\left(D(\mathbf{0}) \leftrightarrow \partial B_{n}\right)^{\mathrm{c}}\right\}$ for $\mathrm{P}_{\lambda \pm \varepsilon}\left(D(\mathbf{0}) \stackrel{\star}{\leftrightarrow} \partial B_{n}\right)$, keeping the inequality signs unchanged, using the previous argument on vacancy.

From Lemma 3.3 of Meester and Roy (1996), it is known that, for $\lambda<\lambda_{\mathrm{c}}$, the diameter of the occupied component of the origin has exponential decay. It then follows that $\phi(\lambda)>0$ for $0<\lambda<\lambda_{\mathrm{c}}$. As $\phi(\lambda)=0$ for $\lambda>\lambda_{\mathrm{c}}$ and as $\phi$ is continuous, we have $\phi\left(\lambda_{\mathrm{c}}\right)=0$. Note that $\mathrm{P}_{\lambda}\left(\mathbf{0} \leftrightarrow \partial B_{n}\right) \leq \mathrm{P}_{\lambda}(|W| \geq n)$, where $|W|$ is the number of Poisson points in the occupied component of the origin. Now $\mathrm{P}_{\lambda}(|W| \geq n) \leq \mathrm{P}_{\lambda}\left(\left|W_{\{\rho=1\}}\right| \geq n\right)$, where the second probability is that of a Poisson Boolean model with constant radius 1 . From Theorem 10.1 of Penrose (2003), it follows that $\lim _{n \rightarrow \infty}-(1 / n) \log \mathrm{P}_{\lambda}\left(\left|W_{\{\rho=1\}}\right| \geq n\right)=\zeta(\lambda) \uparrow \infty$ as $\lambda \downarrow 0$ and, since $\phi(\lambda) \geq \zeta(\lambda)$, we have $\phi(\lambda) \uparrow \infty$ as $\lambda \downarrow 0$.

To show that $\phi(\lambda)$ is strictly decreasing on $\left(0, \lambda_{c}\right)$, we adapt the argument in Grimmett (1983) to our continuum setting. Let $N(k)$ be the number of Poisson $(\lambda)$ points in the component of the origin which fall in the annulus $B_{k} \cap B_{k-1}^{\mathrm{c}}$ and $N(n)=(N(1), N(2), \ldots, N(n))$. Consider another intensity $\lambda^{\prime}, 0<\lambda^{\prime}<\lambda<\lambda_{\mathrm{c}}$. The points with intensity $\lambda$ are called 'light' and each of them can be 'white' with conditional probability $\lambda^{\prime} / \lambda$. Let $A_{n}$ be the event that the origin is joined to $\partial B_{n}$ by a 'white' path. If $\boldsymbol{m}(n)=(m(1), m(2), \ldots, m(n))$ then, writing $\varepsilon=1-\lambda^{\prime} / \lambda$, we have

$$
\mathrm{P}\left(A_{n} \mid \boldsymbol{N}(n)=\boldsymbol{m}(n)\right) \leq \prod_{i=1}^{n}\left(1-\varepsilon^{m(i)}\right) \leq \exp \left(-\sum_{i=1}^{n} \varepsilon^{m(i)}\right),
$$

since each 'light' point is not 'white' with probability $\varepsilon$. Now

$$
\beta_{\lambda^{\prime}}(n)=\mathrm{P}\left(A_{n}\right)=\sum \mathrm{P}\left(A_{n} \mid N(n)\right) \mathrm{P}(\boldsymbol{N}(n)=\boldsymbol{m}(n)),
$$

where the sum is over all vectors $\boldsymbol{m}(n)$ such that the conditional probability is nonzero.

Dividing the sum into two parts depending on whether $\sum_{i=1}^{n} m(i) \leq M n$ or $\sum_{i=1}^{n} m(i)>$ $M n$, where $M$ will be specified later, we obtain

$$
\beta_{\lambda^{\prime}}(n) \leq \sum_{\left\{\boldsymbol{m}: \sum_{i} m(i) \leq M n\right\}} \exp \left(-\sum_{i=1}^{n} \varepsilon^{m(i)}\right) \mathrm{P}(\boldsymbol{N}(n)=\boldsymbol{m}(n))+\mathrm{P}(|L|>M n),
$$

where $|L|$ denotes the number of Poisson points in the 'light' cluster containing the origin.

By the inequality between the arithmetic and geometric means we see that, the first term on the right-hand side of inequality (3.11) is bounded above by

$$
\sum_{\left\{\boldsymbol{m}: \sum_{i} m(i) \leq M n\right\}} \mathrm{e}^{-n \varepsilon^{M}} \mathrm{P}(\boldsymbol{N}(n)=\boldsymbol{m}(n)) \leq \mathrm{e}^{-n \varepsilon^{M}} \beta_{\lambda}(n-1) .
$$

For the second term on the right-hand side of inequality (3.11), we need to do some work.

Lemma 3.1. Let $(X, \lambda, \rho)$ be a Poisson Boolean model on $\mathbb{R}^{d}$ with $0<\rho \leq 1$. Let $C(\mathbf{0})$ denote the occupied cluster of the origin $\mathbf{0}$, and let $\# C(\mathbf{0})$ denote the number of Poisson points of $X$ lying in $C(\mathbf{0})$. For $\lambda<\lambda_{\mathrm{c}}$ and all $n$, we have

$$
\mathrm{P}_{\lambda}(\# C(\mathbf{0})>n) \leq C_{1} \mathrm{e}^{-C_{2} n}
$$

for some positive constants $C_{1}$ and $C_{2}$ which do not depend on $n$. 
Assuming that Lemma 3.1 holds for the moment we complete the proof of Proposition 3.2. Choose $M$ such that $C_{2} M>\phi(\lambda)+\varepsilon^{M}$. Then, taking logarithms of both sides of (3.11) and dividing by $n$, we have, for all sufficiently large $n$,

$$
\begin{aligned}
\frac{1}{n} \log \beta_{\lambda^{\prime}}(n) & \leq \frac{1}{n} \log \left[C_{1} \mathrm{e}^{-C_{2} M n}+\mathrm{e}^{-n \varepsilon^{M}} \beta_{\lambda}(n-1)\right] \\
& \leq \frac{1}{n} \log \left[C_{1} \mathrm{e}^{-n\left[\phi(\lambda)+\varepsilon^{M}\right]}+\mathrm{e}^{-n \varepsilon^{M}} C_{3}(\lambda, \rho)(n-1) \mathrm{e}^{-(n-1) \phi(\lambda)}\right] \\
& \leq-\phi(\lambda)-\varepsilon^{M}+\frac{1}{n} \log \left[C_{1}+C_{3}(\lambda, \rho)(n-1) \mathrm{e}^{\phi(\lambda)}\right],
\end{aligned}
$$

where we have used the bound of $\beta_{\lambda}(n)$ in the second inequality with $C_{3}(\lambda, \rho)=64(1-$ $\left.\mathrm{e}^{-\lambda t^{2} \mathrm{P}(\rho>2 t)}\right)^{-9 / t^{2}}$. Now, letting $n \rightarrow \infty$, we conclude that $\phi\left(\lambda^{\prime}\right) \geq \phi(\lambda)+\varepsilon^{M}$, thereby proving the result.

Proof of Lemma 3.1. First observe that, for $b>0$ and a random variable $\rho_{b}$ defined on the same probability space as that of $\rho$ and whose distribution function is given by $\mathrm{P}\left(\rho_{b} \leq t\right)=$ $\mathrm{P}(\rho \leq t \mid \rho \geq b)$, we have $\rho_{b} \downarrow \rho$ almost surely. Thus, by Theorem 3.7 of Meester and Roy (1996), we have $\lambda_{\mathrm{c}}\left(\rho_{b}\right) \uparrow \lambda_{\mathrm{c}}(\rho)$; so we can choose $b>0$ such that $\lambda<\lambda_{\mathrm{c}}\left(\rho_{b}\right) \leq \lambda_{\mathrm{c}}(\rho)$. Coupling the processes $(X, \lambda, \rho)$ and $\left(X, \lambda, \rho_{b}\right)$ we see that $C(\mathbf{0}) \subseteq C_{b}(\mathbf{0})$, where $C_{b}(\mathbf{0})$ denotes the occupied cluster of the origin $\mathbf{0}$ in $\left(X, \lambda, \rho_{b}\right)$. Thus, we have

$$
\mathrm{P}_{\lambda, \rho}(\# C(\mathbf{0})>n) \leq \mathrm{P}_{\lambda, \rho_{b}}\left(\# C_{b}(\mathbf{0})>n\right) .
$$

Hence, it suffices to prove the lemma for the Boolean model $\left(X, \lambda, \rho_{b}\right)$, where $0<b \leq \rho_{b} \leq 1$.

Fix $\alpha>0$, to be chosen later. Taking $\ell(S)$ to denote the Lebesgue measure of a Borel region $S \subseteq \mathbb{R}^{d}$, we note that

$$
\begin{aligned}
\mathrm{P}_{\lambda}\left(\# C_{b}(\mathbf{0})>n\right)= & \mathrm{P}\left(\# C_{b}(\mathbf{0})>n, \ell\left(C_{b}(\mathbf{0})\right)>\alpha n\right) \\
& +\mathrm{P}_{\lambda}\left(\# C_{b}(\mathbf{0})>n, \ell\left(C_{b}(\mathbf{0})\right) \leq \alpha n\right) .
\end{aligned}
$$

For the first term in the above sum, we have, from Lemma 3.3 and Theorem 3.5 of Meester and Roy (1996), for $\lambda<\lambda_{\mathrm{c}}\left(\rho_{b}\right)$ and some positive constants $C_{1}$ and $C_{2}$,

$$
\mathrm{P}_{\lambda}\left(\# C_{b}(\mathbf{0})>n, \ell\left(C_{b}(\mathbf{0})\right)>\alpha n\right) \leq \mathrm{P}_{\lambda}\left(\ell\left(C_{b}(\mathbf{0})\right)>\alpha n\right) \leq C_{1} \exp \left(-C_{2} \alpha n\right) .
$$

To handle the second term, we need a geometric observation. Let $K$ be a finite set such that

$$
\left[-\frac{3}{2}, \frac{3}{2}\right]^{d} \subset K+\left[-\frac{1}{2} b, \frac{1}{2} b\right]^{d} .
$$

Claim 3.1. Let $x, y \in \mathbb{R}^{d}$ and $r \in[b, 1]$. If $\left(x+\left[-\frac{1}{2}, \frac{1}{2}\right]^{d}\right) \cap\left(y+[-r / 2, r / 2]^{d}\right)$ is nonempty then $\left(x+\left[-\frac{1}{2}, \frac{1}{2}\right]^{d}\right) \subset K+y+[-r / 2, r / 2]^{d}$.

Proof. As $r \leq 1$, if $\left(x+\left[-\frac{1}{2}, \frac{1}{2}\right]^{d}\right) \cap\left(y+[-r / 2, r / 2]^{d}\right)$ is nonempty, then $x-y$ belongs to $[-1,1]^{d}$. Therefore, $\left(x-y+\left[-\frac{1}{2}, \frac{1}{2}\right]^{d}\right) \subset\left[-\frac{3}{2}, \frac{3}{2}\right]^{d}$. By the definition of $K$, we then get $\left(x-y+\left[-\frac{1}{2}, \frac{1}{2}\right]^{d}\right) \subset K+[-b / 2, b / 2]^{d} \subset K+[-r / 2, r / 2]^{d}$. This implies the claim.

Now, we set $D(z)=z+\left[-\frac{1}{2}, \frac{1}{2}\right]^{d}$ for $z \in \mathbb{Z}^{d}$. Let $V_{1}=\left\{z \in \mathbb{Z}^{d}: D(z) \cap C_{b}(\mathbf{0}) \neq \varnothing\right\}$ and $R_{1}=\bigcup_{z \in V_{1}} D(z)$. Clearly, $C_{b}(\mathbf{0}) \subset R_{1}$. By Claim 3.1, we have $R_{1} \subset K+C_{b}(\mathbf{0})$. Hence, $\ell\left(R_{1}\right) \leq(\# K) \ell\left(C_{b}(\mathbf{0})\right)$. Furthermore, since $C_{b}(\mathbf{0})$ is connected, $V_{1}$ (as a subset of $\mathbb{Z}^{d}$ ) is 
$\|\cdot\|_{\infty}$-connected, i.e. for any $z_{1}, z_{2} \in V_{1}$ and some $n \geq 1$, there exists $z_{1}=\boldsymbol{u}_{0}, \boldsymbol{u}_{1}, \ldots, \boldsymbol{u}_{n}=$ $z_{2}$ in $V_{1}$ such that $\left\|\boldsymbol{u}_{i}-\boldsymbol{u}_{i+1}\right\|_{\infty}=1$ for $i=0,1, \ldots, n-1$.

Next, we set $V_{2}=V_{1}+\{-1,0,1\}^{d}$ and $R_{2}=\bigcup_{z \in V_{2}} D(z)$. Clearly, $R_{1} \subset R_{2}$. We have $\ell\left(R_{2}\right) \leq 3^{d} \ell\left(R_{1}\right) \leq 3^{d}(\# K) \ell\left(C_{b}(\mathbf{0})\right)$. Furthermore, we claim that $V_{2}$ (as a subset of $\mathbb{Z}^{d}$ ) is $\|\cdot\|_{1}$-connected, i.e. for any $z_{1}, z_{2} \in V_{2}$ and some $n \geq 1$, there exists $z_{1}=\boldsymbol{u}_{0}, \boldsymbol{u}_{1}, \ldots, \boldsymbol{u}_{n}=\boldsymbol{z}_{2}$ in $V_{2}$ such that $\left\|\boldsymbol{u}_{i}-\boldsymbol{u}_{i+1}\right\|_{1}=1$ for $i=0,1, \ldots, n-1$. Indeed, for $z_{1}, z_{2} \in V_{1}+$ $\{-1,0,1\}^{d}$, we have $z_{1}^{\prime}$ and $z_{2}^{\prime} \in V_{1}$ such that $z_{1} \in z_{1}^{\prime}+\{-1,0,1\}^{d}$ and $z_{2} \in z_{2}^{\prime}+\{-1,0,1\}^{d}$. Furthermore, since $V_{1}$ is $\|\cdot\|_{\infty}$-connected, we have, for some $n \geq 1, z_{1}^{\prime}=\boldsymbol{u}_{0}, \boldsymbol{u}_{1}, \ldots, \boldsymbol{u}_{n}=z_{2}^{\prime}$ in $V_{1}$ such that $\left\|\boldsymbol{u}_{i}-\boldsymbol{u}_{i+1}\right\|_{\infty}=1$ for $i=0,1, \ldots, n-1$. Therefore, it is enough to show that if $\boldsymbol{z} \in \boldsymbol{z}_{0}+\{-1,0,1\}^{d}$ then there exists a sequence $\boldsymbol{z}_{0}=\boldsymbol{v}_{0}, \boldsymbol{v}_{1}, \boldsymbol{v}_{2}, \ldots, \boldsymbol{v}_{m}=\boldsymbol{z}$ for some $m \geq 1$, where $\left\|\boldsymbol{v}_{i}-\boldsymbol{v}_{i+1}\right\|_{1}=1$ for $i=0,1,2, \ldots, m-1$ and $\boldsymbol{v}_{i} \in z_{0}+\{-1,0,1\}^{d}$ for $i=1,2, \ldots, m-1$. This is easily done by changing coordinates one by one, starting from $z_{0}$ and ending at $z$, and ignoring repeated coordinates. More precisely, set $\boldsymbol{v}_{(0)}=z_{0}$ and $\boldsymbol{v}_{(d)}=\boldsymbol{z}$, and, for $1 \leq i \leq d-1$, set

$$
\boldsymbol{v}_{(i)}=\left(z(1), z(2), \ldots, z(i), z_{0}(i+1), z_{0}(i+2), \ldots, z_{0}(d)\right)
$$

where

$$
z=(z(1), z(2), \ldots, z(d)) \quad \text { and } \quad z_{0}=\left(z_{0}(1), z_{0}(2), \ldots, z_{0}(d)\right) .
$$

Clearly, $\left\|\boldsymbol{v}_{(i)}-\boldsymbol{v}_{(i+1)}\right\|_{1} \leq 1$ for $i=0,1, \ldots, d-1$. Set $\tau_{0}=0$ and, for $i \geq 1, \tau_{i+1}=\{k>$ $\left.\tau_{i}:\left\|\boldsymbol{v}_{\left(\tau_{i}\right)}-\boldsymbol{v}_{(k)}\right\|_{1}=1\right\}$. Clearly, for some $m \leq d$, we must have $\boldsymbol{v}_{\left(\tau_{m}\right)}=\boldsymbol{z}$ and $\tau_{m+k}=\infty$ for $k \geq 1$. Finally, choose $\boldsymbol{v}_{i}=\boldsymbol{v}_{\left(\tau_{i}\right)}$ for $i=0,1, \ldots, m$. Thus, if $\ell\left(C_{b}(\mathbf{0})\right) \leq \alpha n$, there exists a connected region $R_{2}$ containing $C_{b}(\mathbf{0})$ and comprising of unit cells of the lattice $\mathbb{Z}^{2}$ such that $\ell\left(R_{2}\right) \leq 3^{d}(\# K) \alpha n$. An upper bound of the number of $\|\cdot\|_{1}$-connected sets $N_{n}$ with at most $3^{d}(\# K) \alpha n$ vertices is given by $\gamma^{3^{d}(\# K) \alpha n}$ for some constant $\gamma>1$ (see, e.g. Grimmett (1999, Equation (4.24))).

Noting that, for both $\left\{\# C_{b}(\mathbf{0})>n\right\}$ and $\left\{\ell\left(C_{b}(\mathbf{0})\right) \leq \alpha n\right\}$ to occur, at least one of the $N_{n}$ connected sets must have $n$ many Poisson points, we have, for a Poisson random variable $Y$ with mean $3^{d}(\# K) \lambda \alpha n$,

$$
\begin{aligned}
\mathrm{P}_{\lambda}(\# C(\mathbf{0})>n, \ell(C(\mathbf{0})) \leq \alpha n) & \leq \gamma^{3^{d}(\# K) \alpha n} \mathrm{P}(Y \geq n) \\
& =\gamma^{3^{d}(\# K) \alpha n} \mathrm{P}\left(\mathrm{e}^{Y} \geq \mathrm{e}^{n}\right) \\
& \leq \gamma^{3^{d}(\# K) \alpha n} \frac{\exp \left(3^{d}(\# K) \lambda \alpha n(\mathrm{e}-1)\right)}{\mathrm{e}^{n}},
\end{aligned}
$$

where the last inequality follows from Markov's inequality. Thus, for $\alpha$ such that

$$
3^{d}(\# K) \alpha\left[\lambda_{\mathrm{c}}(\mathrm{e}-1)+\log \gamma\right]<1,
$$

we have

$$
\begin{aligned}
\mathrm{P}_{\lambda}(\# C(\mathbf{0})>n, \ell(C(\mathbf{0})) \leq \alpha n) & \leq \exp \left(-n\left[1-3^{d}(\# K) \alpha(\lambda(\mathrm{e}-1)+\log \gamma)\right]\right) \\
& \leq C_{5} \exp \left(-C_{6} n\right)
\end{aligned}
$$

for some constants $C_{5}, C_{6}>0$. This completes the proof of Lemma 3.1.

Proof of Theorem 1.2(iii) for an infinite vacant component. Suppose that $\lambda<\lambda_{\mathrm{c}}$, and recall that $\phi(\lambda)=1 / \xi(\lambda)$. When $a<\xi(\lambda)$, then we want to show that $L_{f}$ almost surely contains no 
unbounded vacant cluster. Let $\lambda_{a}^{\star}$ be the unique solution of $\xi(\lambda)=a$. Note that over $\left(0, \lambda_{\mathrm{c}}\right)$, $\xi(\lambda)$ is increasing; thus, $a<\xi(\lambda)$ implies that $\lambda>\lambda_{a}^{\star}$. Fixing $\delta>0$ such that $(1+\delta) a<\xi(\lambda)$, we define $\boldsymbol{w}_{k}=\left(k^{1+\delta}, 0\right)$. Let $B_{k}$ be the smallest square with $\boldsymbol{w}_{k}$ in the middle of the lower side with the upper side just above the curve $v=f(u)$. We have $f(u) / \log u \rightarrow a$; hence, the side length $l_{k}$ of $B_{k}$ satisfies

$$
l_{k}=a(1+o(1)) \log k^{1+\delta} \quad \text { as } k \rightarrow \infty .
$$

Now $B_{k}$ has side length $l_{k}$ and centre $\boldsymbol{w}_{k}+\left(0, l_{k} / 2\right)$. Let $A_{k}$ be the event that there is an occupied path from the top edge to the bottom edge of $B_{k}$. By the FKG inequality,

$$
\mathrm{P}_{\lambda}\left(A_{k}\right) \geq\left\{\frac{1}{4} \mathrm{P}_{\lambda}\left(D(\mathbf{0}) \leftrightarrow \partial B_{k}\left(\frac{1}{2} l_{k}\right)\right)\right\}^{2}\left(1-\mathrm{e}^{-\lambda t^{2} \mathrm{P}(\rho>2 t)}\right)^{9 / t^{2}},
$$

where $t$ was defined at the beginning of this subsection satisfying $\mathrm{P}(\rho>2 t)>0$. However,

$$
\mathrm{P}_{\lambda}\left(D(\mathbf{0}) \leftrightarrow \partial B_{k}\left(\frac{1}{2} l_{k}\right)\right) \approx \mathrm{e}^{-l_{k}(2 \xi(\lambda))^{-1}} \quad \text { as } k \rightarrow \infty,
$$

by (3.10). Hence,

$$
\mathrm{P}_{\lambda}\left(A_{k}\right) \geq \frac{1}{16} k^{-(1+o(1))(1+\delta) a / \xi(\lambda)}\left(1-\mathrm{e}^{-\lambda t^{2} \mathrm{P}(\rho>2 t)}\right)^{9 / t^{2}}
$$

as $k \rightarrow \infty$, i.e. $\sum \mathrm{P}_{\lambda}\left(A_{k}\right)=\infty$ since $(1+\delta) a<\xi(\lambda)$. On the other hand, the squares $B_{k}$ are separated by more than twice the maximum of the sides of the Boolean squares (assumed that $R=1$ here); hence, the configurations inside the $B_{k}$ s are independent for large $k$. Thus, $A_{k}$ occurs infinitely often almost surely.

Secondly, suppose that $\lambda<\lambda_{\mathrm{c}}$ and $a>\xi(\lambda)$. We want to show that $L_{f}$ almost surely contains an infinite vacant cluster. Choose $\alpha$ such that $a>\alpha>\xi(\lambda)$, and let $D_{k}$ be the box with centre $(k, 0)$ and side length $2 \alpha \log k$. For large values of $k, D_{k}$ lies strictly beneath the curve $v=f(u)$. Let $E_{k}$ be the event that $(k, 0)$ is joined by an occupied path to $\partial D_{k}$. From (3.10) we have

$$
\mathrm{P}_{\lambda}\left(E_{k}\right)=\mathrm{P}_{\lambda}(\mathbf{0} \leftrightarrow \partial B(\alpha \log k)) \leq \mathrm{P}_{\lambda}(D(\mathbf{0}) \leftrightarrow \partial B(\alpha \log k)) \approx k^{-\alpha / \xi(\lambda)}
$$

as $k \rightarrow \infty$. This gives $\sum \mathrm{P}_{\lambda}\left(E_{k}\right)<\infty$ from the assumption that $\alpha>\xi(\lambda)$. Therefore, there exists an $M$ such that

$$
\mathrm{P}_{\lambda}\left(\bigcup_{k \geq M} E_{k}\right)<\frac{1}{2} .
$$

However, if none of the events $\left\{E_{k}, k \geq M\right\}$ occurs then an occupied path cannot join $f(u)$ and $\mathbb{R}^{+}$, and $L_{f}$ almost surely contains an infinite vacant cluster.

Combining the above proves that $\lambda_{\mathrm{c}}^{\star}\left(L_{f}\right)$ is the unique solution in $\left(0, \lambda_{\mathrm{c}}\left(\mathbb{R}^{2}\right)\right)$ of $\xi(\lambda)=a$.

Proof of Theorem 1.2(i) and (ii) for an infinite vacant component. In (i), $\lambda_{\mathrm{c}}^{\star}\left(L_{f}\right)=0$ follows from the fact that the solution of $\xi(\lambda)=a$ goes to 0 as $a \downarrow 0$. Similarly, in (ii), $\lambda_{\mathrm{c}}^{\star}\left(L_{f}\right)=\lambda_{\mathrm{c}}\left(\mathbb{R}^{2}\right)$ follows from the fact that the solution of $\xi(\lambda)=a$ goes to $\lambda_{\mathrm{c}}\left(\mathbb{R}^{2}\right)$ as $a \uparrow \infty$.

Remark. At criticality, i.e. when $f(x) \sim a \log x$ for some $a>0$ and $\lambda=\lambda_{\mathrm{c}}\left(L_{f}\right)$ as obtained in Theorem 1.2(iii), an infinite occupied component is possible and, similarly, at $\lambda_{\mathrm{c}}^{\star}\left(L_{f}\right)$ an infinite vacant component is possible. Let us consider the case of $\lambda_{\mathrm{c}}\left(L_{f}\right)$ and show that there exists a function $f$ such that $f(x) / \log x \rightarrow a$ as $a \rightarrow \infty$ with $\xi^{\star}\left(\lambda_{a}\right)=a$. We have

$$
\mathrm{P}_{\lambda_{a}}\left(L_{f} \text { contains an infinite occupied cluster }\right)=1 \text {. }
$$


Consider the function $f$ satisfying $f(u)=a \log u+b \log \log u$ for all large $u$ where $b>2 a$. Let $D_{k}$ be the largest box having centre at $(k, 0)$ and lying strictly beneath the curve $v=f(u)$. Then $D_{k}$ has side length $2 f(k)+O(1)$ as $k \rightarrow \infty$. Let $E_{k}$ be the event that $(k, 0)$ is joined by a vacant path to $\partial D_{k}$. Instead of (3.8), $\mathrm{P}_{\lambda}\left(E_{k}\right)$ can be bounded more precisely by the inequality on the right-hand side of (3.1) as

$$
\begin{aligned}
\mathrm{P}_{\lambda}\left(E_{k}\right) & \leq \mathrm{P}_{\lambda}(D(\mathbf{0}) \stackrel{\star}{\leftrightarrow} \partial B(f(k)+O(1))) \\
& \leq 64 \mathrm{e}^{9 \lambda}[f(k)+O(1)] \exp \left(-\frac{a \log k+b \log \log k}{\xi^{\star}(\lambda)}\right) \\
& \leq 128 a \mathrm{e}^{9 \lambda}(\log k) \exp \left(-\frac{a \log k+b \log \log k}{\xi^{\star}(\lambda)}\right)
\end{aligned}
$$

for all large $k$. At $\lambda_{a}$ we have $\xi^{\star}\left(\lambda_{a}\right)=a$ and then $\mathrm{P}_{\lambda_{a}}\left(E_{k}\right) \leq C k^{-1}(\log k)^{-\alpha}$, where $C=$ $128 a \mathrm{e}^{9 \lambda}$ and $\alpha=b / a-1>1$. Summability of $\mathrm{P}_{\lambda_{a}}\left(E_{k}\right)$ as before gives (3.12), following the argument after (3.9). The calculations for $\lambda_{a}^{\star}$ are similar.

\section{Acknowledgements}

We thank the anonymous referees for a careful reading of the paper and suggestions for improvement. We also thank them for the simple proof of the claim in Section 3.2.

\section{References}

Athreya, S., Roy, R. And Sarkar, A. (2004). On the coverage of space by random sets. Adv. Appl. Prob. 36, 1-18.

Grimmett, G. R. (1983). Bond percolation on subsets of the square lattice, and the threshold between one-dimensional and two-dimensional behaviour. J. Phys. A. 16, 599-604.

Grimmett, G. R. (1999). Percolation, 2nd edn. Springer, Berlin.

Hall, P. (1988). Introduction to the Theory of Coverage Processes. John Wiley, New York.

Meester, R. ANd Roy, R. (1996). Continuum Percolation. Cambridge University Press.

Molchanov, I. and Scherbakov, V. (2003). Coverage of the whole space. Adv. Appl. Prob. 35, 898-912.

Penrose, M. (2003). Random Geometric Graphs. Oxford University Press.

Petrov, V. V. (2004). A generalization of the Borel-Cantelli lemma. Statist. Prob. Lett. 67, 233-239.

Stoyan, D., Kendall, W. S. And Mecke, J. (1987). Stochastic Geometry and Its Applications. John Wiley, Chichester. Tanemura, H. (1993). Behavior of the supercritical phase of a continuum percolation model on $\mathbb{R}^{d}$. J. Appl. Prob. 30, 382-396.

Tanemura, H. (1996). Critical behavior for a continuum percolation model. In Probability Theory and Mathematical Statistics. (Tokyo, 1995), World Scientific, River Edge, NJ, pp. 485-495. 